\title{
A Serre-type Theorem for the Elliptic Lie Algebras with Rank $\geq 2$
}

By

\author{
Hiroyuki YAMANE*
}

\begin{abstract}
In 2000, K. Saito and D. Yoshii gave a Serre-type theorem for the simply-laced elliptic Lie algebras. We extend the theorem to that for the elliptic Lie algebras associated with the (reduced marked) elliptic root systems with rank $\geq 2$.
\end{abstract}

\section{Introduction}

In the early eighties, K. Saito [S] introduced the concept of the generalized root systems and, in particular, the elliptic root systems. Since then, several attempts have been done to construct Lie algebras having the property that their "real roots" form those root systems (see [SY, Introduction]). In the final year of the last century, K. Saito and D. Yoshii [SY] introduced three kinds of "universal" presentations of the simply-laced elliptic Lie algebras $\mathfrak{g}(R)$, that is, the elliptic Lie algebras associated with the simply-laced elliptic root systems $R$. We can say that the $\mathfrak{g}(R)$ is maximal among the Lie algebras having the above property (see also the second paragraph). Let us explain the presentations. The first one uses the Borcherds lattice vertex algebras. This can be said to be most beautiful and useful, because it does not depend on a marking $G$ of $R$ and gives a basis of $\mathfrak{g}(R)$ and its structure constants explicitly. The second one uses (affine-type) Heisenberg algebras. This is also useful, especially to study the representation theory of $\mathfrak{g}(R)$ since it gives a triangular decomposition of $\mathfrak{g}(R)$. The third one is a Serre-type theorem, that is to say a presentation of

Communicated by K. Saito. Received May 9, 2002.

2000 Mathematics Subject Classification(s): Primary 17B65; Secondary 22E65

* Department of Pure and Applied Mathematics, Graduate School of Information Science and Technology, Osaka University, Toyonaka 560-0043, Japan.

e-mail: yamane@ist.osaka-u.ac.jp 
$\mathfrak{g}(R)$ by finite defining relations, which are expressed by means of the elliptic diagram $\Gamma(R, G)$, a Dynkin diagram introduced by K. Saito $[\mathrm{S}]$.

Let $R$ be an elliptic root system; more precisely, $(R, G)$ is assumed to be a reduced marked elliptic root system for some $G$. D. Yoshii $[\mathrm{Y}]$ generalized the second one to present the elliptic Lie algebras $\mathfrak{g}(R)$. We shall do the same as the third one to present $\mathfrak{g}(R)$ in the case of $l \geq 2$, where $l$ is the rank of $R$ (see Theorem 4.1). We also show that if $l \geq 2$ and $\Gamma(R, G) \neq A_{l}^{(1,1)}, \mathfrak{g}(R)$ is maximal among the Lie algebras having the property mentioned above and satisfying extra conditions (see Theorem 3.2). K. Saito and T. Takebayashi [ST] have asked for such presentation as ours. We also notice results given in [M1, M2].

The definitions of some of the above terms shall be given in Appendix.

Now, let us explain about the content of the paper more concretely. We abbreviate the terminology "a reduced marked elliptic root system" to an r.m.e.root-system (see Appendix). Let $l$ be a fixed positive integer. Let $\mathcal{E}$ be an $l+4$-dimensional $\mathbb{C}$-vector space with a non-degenerate symmetric bilinear form $I: \mathcal{E} \times \mathcal{E} \rightarrow \mathbb{C}$. If $x \in \mathcal{E}$ satisfies $I(x, x) \neq 0$, then we call it non-isotropic, let $x^{\vee}:=2 x / I(x, x)$ and define $s_{x} \in \operatorname{GL}(\mathcal{E})$ by $s_{x}(y)=y-I\left(x^{\vee}, y\right) x$. Let $\Pi$ be a subset of $\mathcal{E}$ formed by linearly independent non-isotropic $l+1$ elements satisfying the condition that the $(l+1) \times(l+1)$ matrix formed by the components $I\left(\alpha^{\vee}, \beta\right)$, where $\alpha, \beta \in \Pi$, coincides with an affine-type generalized Cartan matrix. (See $[\mathrm{K}, \S 4.8]$ for the terminology.) Denote by $W$ the affine Weyl group $\left\langle s_{\alpha} \mid \alpha \in \Pi\right\rangle \subset \operatorname{GL}(\mathcal{E})$. Let $a$ be a fixed non-zero element of $\mathcal{E}$ with $\operatorname{dim}(\mathbb{C} a+$ $\mathbb{C} \Pi)=l+2$ and $I(a, \mathbb{C} a+\mathbb{C} \Pi)=\{0\}$. Let $k: \Pi \rightarrow \mathbb{N}$ be a function such that G.C.D. $\{k(\alpha) \mid \alpha \in \Pi\}=1$, and $k(\alpha)=k(\beta)$ if $\beta \in W$. $\alpha$. Let $G:=\mathbb{C} a$ and

$$
R:=\bigcup_{w \in W} \bigcup_{\alpha \in \Pi}(w(\alpha)+\mathbb{Z} k(\alpha) a) .
$$

We see that $(R, G)$ is an r.m.e.-root-system if and only if

$$
\forall \mu \in R, \quad s_{\mu}(R)=R .
$$

If this is the case, we call $k$ the counting function. K. Saito [S] gave all the counting functions concretely and showed that every r.m.e.-root-system is given in this way. (Notice that $l$ is the rank of $R$.)

We say that a triple $(\mathcal{E}, \Pi, k)$ satisfying the property $(0.2)$ is a reduced marked elliptic datum; we abbreviate the terminology to an r.m.e.-datum. (In the text, an r.m.e.-datum $(\mathcal{E}, \Pi, k)$ shall be denoted as $\left(\mathcal{E}_{\mathrm{af}}^{\natural}, \Pi_{\mathrm{af}}, k\right)$, and the $\mathfrak{g}(R)$ shall be denoted as $\mathfrak{g}=\mathfrak{g}\left(\mathcal{E}_{\mathrm{af}}^{\natural}, \Pi_{\mathrm{af}}, k\right)$.)

In this paper, we shall give Lie algebras $\mathfrak{g}^{\natural}, \mathfrak{g}^{\triangle}$ and $\mathfrak{g}^{\Gamma(R, G)}$ and show that all of them are isomorphic to $\mathfrak{g}(R)$ (see Theorems 1.1, 3.1 and 4.1). These Lie 
algebras are virtually the same, but we use these different symbols to make the paper easier to read. Here we give the definition of $\mathfrak{g}^{\natural}$ and explain about how $\mathfrak{g}^{\triangle}$ and $\mathfrak{g}^{\Gamma(R, G)}$ are obtained from $\mathfrak{g}^{\natural}$. Let $(\mathcal{E}, \Pi, k)$ be an r.m.e.-datum with $l \geq 2$. Let $\alpha^{*}:=\alpha+k(\alpha) a$ for $\alpha \in \Pi$. Let $\Pi^{*}:=\left\{\alpha^{*} \mid \alpha \in \Pi\right\}$. Let $\mathcal{B}:=\left\{ \pm \mu \mid \mu \in \Pi \cup \Pi^{*}\right\}$. Define the Lie algebra $\mathfrak{g}^{\natural}$ with generators:

$$
h_{\sigma}(\sigma \in \mathcal{E}), E_{\mu}(\mu \in \mathcal{B})
$$

and defining relations:

$$
\begin{cases}x h_{\sigma}+y h_{\tau}=h_{x \sigma+y \tau} & \text { for } x, y \in \mathbb{C} \text { and } \sigma, \tau \in \mathcal{E}, \\ {\left[h_{\sigma}, h_{\tau}\right]=0} & \text { for } \sigma, \tau \in \mathcal{E}, \\ {\left[h_{\sigma}, E_{\mu}\right]=I(\sigma, \mu) E_{\mu}} & \text { for } \sigma \in \mathcal{E} \text { and } \mu \in \mathcal{B}, \\ {\left[E_{\mu}, E_{-\mu}\right]=h_{\mu^{\vee}}} & \text { for } \mu \in \mathcal{B}, \\ \left(\operatorname{ad} E_{\mu}\right)^{x}{ }_{\mu, \nu} E_{\nu}=0 & \text { for } \mu, \nu \in \mathcal{B} \text { with } \mu+\nu \neq 0, \\ \left(\operatorname{ad} E_{ \pm \alpha^{*}}\right)^{y} E_{ \pm \beta}=\left(\operatorname{ad} E_{ \pm \alpha}\right)^{y} E_{ \pm \beta^{*}} & \text { for }(\alpha, \beta, y) \in \mathcal{A}, \\ \left(\operatorname{ad} E_{ \pm \alpha}\right)^{i}\left(\operatorname{ad} E_{ \pm \alpha^{*}}\right)^{y-i} E_{ \pm \beta}=0 & \text { for }(\alpha, \beta, y) \in \mathcal{A} \text { and } 1 \leq i \leq y-1,\end{cases}
$$

where $x_{\mu, \nu}:=\min \{n \in \mathbb{N} \mid n \mu+\nu \notin R \cup G\}$ and $\mathcal{A}:=\{(\alpha, \beta, y) \in \Pi \times \Pi \times \mathbb{N} \mid \alpha \neq$ $\beta, I(\alpha, \beta) \neq 0, k(\alpha) y=k(\beta)\}$.

As a matter of the fact, some of (0.4) are redundant. We shall define $\mathfrak{g}^{\triangle}$ by the same generators as $(0.3)$ and the defining relations which seem to be necessary ones of (0.4).

K. Saito $[\mathrm{S}]$ introduced the elliptic diagram $\Gamma(R, G)$, which is drawn by use of a subset of $\Pi \cup \Pi^{*}$. The subset is called the elliptic root basis and also denoted by $\Gamma(R, G)$. We shall define the $\mathfrak{g}^{\Gamma(R, G)}$ by the generators $h_{\sigma}(\sigma \in \mathcal{E})$, $E_{ \pm \mu}(\mu \in \Gamma(R, G))$ and defining relations, which are obtained from those of $\mathfrak{g}^{\triangle}$ by adding some additional relations.

In $\S 1$, we shall prove that $\mathfrak{g}^{\triangle} \cong \mathfrak{g}(R)$ using properties of rank two affine Lie algebras. In $\S 2$, we shall prove the properties. In $\S 3$, we shall show that $\mathfrak{g}^{\natural} \cong \mathfrak{g}^{\triangle}$ and show a maximality of $\mathfrak{g}^{\natural}$ except for $A_{l}^{(1,1)}$. In $\S 4$, we shall show that $\mathfrak{g}^{\Gamma(R, G)} \cong \mathfrak{g}^{\triangle}$.

\section{$\S 1$. Isomorphism from $\mathfrak{g}$ to $\mathfrak{g}^{\triangle}$}

Let $l$ be a positive integer. Let $\mathcal{E}_{\text {af }}$ be an $l+2$ dimensional $\mathbb{C}$ vector space with a non-degenerate symmetric bilinear form $I: \mathcal{E}_{\text {af }} \times \mathcal{E}_{\text {af }} \rightarrow \mathbb{C}$. Let $\Pi_{\mathrm{af}}=\left\{\alpha_{0}, \alpha_{1}, \ldots, \alpha_{l}\right\}$ be a set of linearly independent $l+1$ elements of $\mathcal{E}_{\mathrm{af}}$ satisfying the condition that: 
(1) $I\left(\alpha_{i}, \alpha_{i}\right)$ is a positive integer for any $0 \leq i \leq l$,

(2) The $(l+1) \times(l+1)$ matrix $A:=\left(a_{i j}\right)$, where $a_{i j}:=2 I\left(\alpha_{i}, \alpha_{j}\right) / I\left(\alpha_{i}, \alpha_{i}\right)$, is a generalized Cartan matrix of affine type (See $[\mathrm{K}, \S 4.8]$ for the terminology), i.e., it satisfies the condition that there exists a unique $x={ }^{t}\left(x_{0}, \ldots, x_{l}\right) \in \mathbb{Z}_{+}^{l+1}$ such that $\left\{v \in \mathbb{Z}_{+}^{l+1} \mid A v=0\right\}=\mathbb{Z}_{+} x$.

We call the pair $\left(\mathcal{E}_{\mathrm{af}}, \Pi_{\mathrm{af}}\right)$ the affine datum. The element $\delta:=x_{0} \alpha_{0}+\cdots+$ $x_{l} \alpha_{l}$ is called the lowest positive null root, where $x_{i}$ 's are the above integers. Let $\Gamma_{\mathrm{af}}\left(\mathcal{E}_{\mathrm{af}}, \Pi_{\mathrm{af}}\right)$ be the Dynkin diagram of the affine datum $\left(\mathcal{E}_{\mathrm{af}}, \Pi_{\mathrm{af}}\right)$; we use the same definition for the Dynkin diagrams as in [K, $\S 4.8]$; in $[\mathrm{K}, \S 4.8$ TABLE Aff $1,2,3]$, the Dynkin diagrams $\Gamma_{\mathrm{af}}\left(\mathcal{E}_{\mathrm{af}}, \Pi_{\mathrm{af}}\right)$ 's of the affine data $\left(\mathcal{E}_{\mathrm{af}}, \Pi_{\mathrm{af}}\right)$ 's are listed, and they are named:

$$
\left\{\begin{array}{c}
A_{l}^{(1)}(l \geq 1), B_{l}^{(1)}(l \geq 3), C_{l}^{(1)}(l \geq 2), D_{l}^{(1)}(l \geq 4) \\
G_{2}^{(1)}(l=2), F_{4}^{(1)}(l=4), E_{l}^{(1)}(l=6,7,8) \\
A_{2 l}^{(2)}(l \geq 1), A_{2 l-1}^{(2)}(l \geq 3), D_{l+1}^{(2)}(l \geq 2), E_{6}^{(2)}(l=4) \\
D_{4}^{(3)}(l=2)
\end{array}\right.
$$

We also use the same names as above. If $\Gamma_{\mathrm{af}}\left(\mathcal{E}_{\mathrm{af}}, \Pi_{\mathrm{af}}\right)$ is $X_{n}^{(t)}$, one of (1.1), we say that $\left(\mathcal{E}_{\mathrm{af}}, \Pi_{\mathrm{af}}\right)$ is of type $X_{n}^{(t)}$. We follow the same numbering as in $[\mathrm{K}, \S 4.8]$ for the vertices of the $\Gamma_{\mathrm{af}}\left(\mathcal{E}_{\mathrm{af}}, \Pi_{\mathrm{af}}\right)$. Let $\Pi_{\mathrm{af}, \mathrm{fi}}:=\left\{\alpha_{i} \mid 1 \leq i \leq l\right\}$ and $\mathcal{E}_{\text {af, fi }}:=\mathbb{C} \alpha_{1} \oplus \cdots \oplus \mathbb{C} \alpha_{l}$. Then $\mathcal{E}_{\text {af }}=\mathcal{E}_{\text {af, fi }} \oplus \mathbb{C} \delta \oplus \mathbb{C} \Lambda_{\delta}$, where $\Lambda_{\delta} \in \mathcal{E}_{\text {af }}$ is such that $I\left(\Lambda_{\delta}, \mathcal{E}_{\text {af, fi }}\right)=\{0\}, I\left(\Lambda_{\delta}, \delta\right)=1$ and $I\left(\Lambda_{\delta}, \Lambda_{\delta}\right)=0$.

For each affine datum $\left(\mathcal{E}_{\text {af }}, \Pi_{\text {af }}=\left\{\alpha_{0}, \ldots, \alpha_{l}\right\}\right)$, we define the $\mathbb{C}$ vector space $\mathcal{E}_{\text {af }}^{\natural}$ to be $\mathcal{E}_{\text {af }} \oplus \mathbb{C} a \oplus \mathbb{C} \Lambda_{a}$, and extend the symmetric bilinear form $I($, on $\mathcal{E}_{\text {af }}$ to the one on $\mathcal{E}_{\text {af }}^{\natural}$ by $I\left(\mathcal{E}_{\text {af }}^{\natural}, \mathbb{C} a \oplus \mathbb{C} \Lambda_{a}\right)=\{0\}, I(a, a)=I\left(\Lambda_{a}, \Lambda_{a}\right)=0$, $I\left(a, \Lambda_{a}\right)=1$. Let $k: \Pi_{\mathrm{af}} \cup-\Pi_{\mathrm{af}} \rightarrow\{1,2,3,4\}$ be a function satisfying the following properties:

(1) $k(-\alpha)=k(\alpha)$,

(2) there exists an $\alpha$ such that $k(\alpha)=1$,

(3) if $I(\alpha, \alpha) \leq I(\beta, \beta)$ and $I(\alpha, \beta) \neq 0$, then $k(\alpha) \leq k(\beta)$ and $I(\beta, \beta) k(\alpha)$ $=I(\alpha, \alpha) k(\beta) c$ for some $c \in\{1,2,3,4\}$.

We call the triple $\left(\mathcal{E}_{\mathrm{af}}^{\natural}, \Pi_{\mathrm{af}}, k\right)$ a reduced marked elliptic datum; we abbreviate this terminology to an r.m.e.-datum. K. Saito [S] showed that a function $k^{\prime}$ : $\Pi_{\mathrm{af}} \rightarrow\{1,2,3,4\}$ satisfies the properties (1), (2) and (3) above with $k^{\prime}$ in place of $k$ if and only if $k^{\prime}$ is a counting function (see Introduction). In particular, 
the definition of an r.m.e.-datum given here and the one given in Introduction are equivalent.

Definition 1.1 (See also $[\mathrm{Y}])$. Let $\left(\mathcal{E}_{\mathrm{af}}^{\natural}, \Pi_{\mathrm{af}}, k\right)$ be an r.m.e.-datum. (1) For $\sigma \in \mathcal{E}_{\text {af }}^{\natural}$ with $I(\sigma, \sigma) \neq 0$, let $\sigma^{\vee}:=2 \sigma / I(\sigma, \sigma) \in \mathcal{E}_{\text {af }}^{\natural}$. For $\sigma, \tau \in \mathcal{E}_{\text {af }}^{\natural}$, let

$$
J\left(\sigma^{\vee}, \tau\right):= \begin{cases}I\left(\sigma^{\vee}, \tau\right) & \text { if } I\left(\sigma^{\vee}, \tau\right) \in-\mathbb{Z}_{+} \text {and } I(\sigma, \sigma) \neq 0, \\ 0 & \text { otherwise. }\end{cases}
$$

(2) For the above $\left(\mathcal{E}_{\text {af }}^{\natural}, \Pi_{\text {af }}, k\right)$, we define a Lie algebra $\mathfrak{g}=\mathfrak{g}\left(\mathcal{E}_{\text {af }}^{\natural}, \Pi_{\text {af }}, k\right)$ by generators:

$$
Z, h_{\sigma}\left(\sigma \in \mathcal{E}_{\mathrm{af}}^{\natural}\right), E_{\alpha}\left(\alpha \in \Pi_{\mathrm{af}} \cup-\Pi_{\mathrm{af}}\right), H_{\alpha^{\vee}}^{(i)}\left(\alpha \in \Pi_{\mathrm{af}} \cup-\Pi_{\mathrm{af}}, i \in \mathbb{Z}\right)
$$

and relations:

$$
\begin{aligned}
& x h_{\sigma}+y h_{\tau}=h_{x \sigma+y \tau}, \quad\left[h_{\sigma}, h_{\tau}\right]=0, \\
& {\left[h_{\sigma}, E_{\alpha}\right]=I(\sigma, \alpha) E_{\alpha}, \quad\left[h_{\sigma}, H_{\alpha^{\vee}}^{(i)}\right]=I(i a, \sigma) k(\alpha) H_{\alpha^{\vee}}^{(i)},} \\
& \left(\operatorname{ad} E_{\alpha}\right)^{1-J\left(\alpha^{\vee}, \beta\right)} E_{\beta}= \begin{cases}h_{-\alpha \vee} & \text { if } \alpha+\beta=0, \\
0 & \text { otherwise, }\end{cases} \\
& Z=h_{a}, \quad H_{\alpha^{\vee}}^{(0)}=h_{\alpha^{\vee}}, \quad H_{-\alpha^{\vee}}^{(i)}=-H_{\alpha^{\vee}}^{(i)}, \\
& {\left[H_{\alpha^{\vee}}^{(i)}, H_{\beta^{\vee}}^{(j)}\right]= \begin{cases}i k(\alpha) I\left(\alpha^{\vee}, \beta^{\vee}\right) Z & \text { if } i k(\alpha)+j k(\beta)=0, \\
0 & \text { otherwise, }\end{cases} } \\
& {\left[H_{\alpha^{\vee}}^{(i)}, E_{\beta}\right]= \begin{cases}\frac{1}{2} I\left(\alpha^{\vee}, \beta\right)\left[H_{\beta^{\vee}}^{(j)}, E_{\beta}\right] & \text { if } i k(\alpha)=j k(\beta), \\
0 & \text { if } i k(\alpha) \notin k(\beta) \mathbb{Z},\end{cases} } \\
& {\left[H_{\alpha^{\vee}}^{(i)},\left[H_{\alpha^{\vee}}^{(j)}, E_{\alpha}\right]\right]=2\left[H_{\alpha^{\vee}}^{(i+j)}, E_{\alpha}\right], \quad\left[\left[H_{\alpha^{\vee}}^{(i)}, E_{\alpha}\right], E_{-\alpha}\right]=-2 H_{\alpha^{\vee}}^{(i)},} \\
& {\left[\left[H_{\alpha^{\vee}}^{(i)}, E_{\alpha}\right], E_{\beta}\right]=0 \quad \text { if } I(\alpha, \beta) \geq 0,}
\end{aligned}
$$

where $\sigma, \tau \in \mathcal{E}_{\mathrm{af}}^{\natural}, x, y \in \mathbb{C}, i, j \in \mathbb{Z}$ and $\alpha, \beta \in \Pi_{\mathrm{af}} \cup-\Pi_{\mathrm{af}}$.

Here, for an r.m.e.-datum $\left(\mathcal{E}_{\mathrm{af}}^{\natural}, \Pi_{\mathrm{af}}, k\right)$ with $l \geq 2$, we introduce an algebra presented by finite generators and finite relations.

Definition 1.2. Let $\left(\mathcal{E}_{\mathrm{af}}^{\natural}, \Pi_{\mathrm{af}}, k\right)$ be an r.m.e.-datum. Assume $l \geq 2$.

(1) For $\alpha \in \Pi_{\mathrm{af}}$, let $\alpha^{*}:=\alpha+k(\alpha) a \in \mathcal{E}_{\mathrm{af}}^{\natural}$.

(2) For the above $\left(\mathcal{E}_{\text {af }}^{\natural}, \Pi_{\text {af }}, k\right)$, we define a Lie algebra $\mathfrak{g}^{\triangle}=\mathfrak{g}^{\triangle}\left(\mathcal{E}_{\text {af }}^{\natural}, \Pi_{\text {af }}, k\right)$ by generators:

$$
h_{\sigma}\left(\sigma \in \mathcal{E}_{\mathrm{af}}^{\natural}\right), E_{\alpha}, E_{-\alpha}, E_{\alpha^{*}}, E_{-\alpha^{*}}\left(\alpha \in \Pi_{\mathrm{af}}\right),
$$


and relations:

$$
\begin{aligned}
& x h_{\sigma}+y h_{\tau}=h_{x \sigma+y \tau}(x, y \in \mathbb{C}), \quad\left[h_{\sigma}, h_{\tau}\right]=0, \\
& \left\{\begin{array}{l}
{\left[h_{\sigma}, E_{\alpha}\right]=I(\sigma, \alpha) E_{\alpha},\left[h_{\sigma}, E_{\alpha^{*}}\right]=I\left(\sigma, \alpha^{*}\right) E_{\alpha^{*}},} \\
{\left[h_{\sigma}, E_{-\alpha}\right]=-I(\sigma, \alpha) E_{-\alpha},\left[h_{\sigma}, E_{-\alpha^{*}}\right]=-I\left(\sigma, \alpha^{*}\right) E_{-\alpha^{*}},}
\end{array}\right. \\
& \left\{\begin{array}{l}
{\left[E_{\alpha}, E_{-\alpha}\right]=-h_{\alpha^{\vee}},\left[E_{\alpha^{*}}, E_{-\alpha^{*}}\right]=-h_{\left(\alpha^{*}\right)^{\vee},}} \\
{\left[E_{\alpha}, E_{\alpha^{*}}\right]=0,\left[E_{-\alpha}, E_{-\alpha^{*}}\right]=0,} \\
\left(\operatorname{ad} E_{\alpha}\right)^{3} E_{-\alpha^{*}}=0,\left(\operatorname{ad} E_{-\alpha}\right)^{3} E_{\alpha^{*}}=0, \\
\left(\operatorname{ad} E_{\alpha^{*}}\right)^{3} E_{-\alpha}=0,\left(\operatorname{ad} E_{-\alpha^{*}}\right)^{3} E_{\alpha}=0,
\end{array}\right. \\
& \left\{\begin{array}{l}
{\left[E_{\alpha}, E_{-\beta}\right]=0,\left[E_{\alpha}, E_{-\beta^{*}}\right]=0, \quad\left[E_{\alpha^{*}}, E_{-\beta^{*}}\right]=0,} \\
\left(\operatorname{ad} E_{\alpha}\right)^{1-I\left(\alpha^{\vee}, \beta\right)}\left(E_{\beta}\right)=0,\left(\operatorname{ad} E_{-\alpha}\right)^{1-I\left(\alpha^{\vee}, \beta\right)}\left(E_{-\beta}\right)=0, \\
\left(\operatorname{ad} E_{\alpha}\right)^{1-I\left(\alpha^{\vee}, \beta\right)}\left(E_{\beta^{*}}\right)=0,\left(\operatorname{ad} E_{-\alpha}\right)^{1-I\left(\alpha^{\vee}, \beta\right)}\left(E_{-\beta^{*}}\right)=0 \\
\text { for } \alpha, \beta \in \Pi_{\mathrm{af}} \text { with } \alpha \neq \beta,
\end{array}\right. \\
& \left\{\begin{array}{l}
\left(\operatorname{ad} E_{\alpha^{*}}\right)^{y} E_{\beta}=\left(\operatorname{ad} E_{\alpha}\right)^{y} E_{\beta^{*}}, \\
\left(\operatorname{ad} E_{-\alpha^{*}}\right)^{y} E_{-\beta}=\left(\operatorname{ad} E_{-\alpha}\right)^{y} E_{-\beta^{*}} \\
\text { for } \alpha, \beta \in \Pi_{\mathrm{af}} \text { with } I(\alpha, \beta) \neq 0 \text { and } I(\alpha, \alpha) \leq I(\beta, \beta), \\
\text { where } y:=k(\beta) / k(\alpha),
\end{array}\right. \\
& \left\{\begin{array}{l}
\left(\operatorname{ad} E_{\beta^{*}}\right)^{2} E_{\alpha}=0,\left(\operatorname{ad} E_{-\beta^{*}}\right)^{2} E_{-\alpha}=0 \\
\text { for } \alpha, \beta \in \Pi_{\text {af }} \text { with } I\left(\beta^{\vee}, \alpha\right)=-1 \text { and } k(\beta)=k(\alpha),
\end{array}\right. \\
& \left\{\begin{array}{l}
\left(\operatorname{ad} E_{\alpha^{*}}\right)^{3} E_{\beta}=0,\left[E_{\alpha},\left[E_{\alpha^{*}}, E_{\beta}\right]\right]=0, \\
\left(\operatorname{ad} E_{-\alpha^{*}}\right)^{3} E_{-\beta}=0,\left[E_{-\alpha},\left[E_{-\alpha^{*}}, E_{-\beta}\right]\right]=0 \\
\text { for } \alpha, \beta \in \Pi_{\text {af }} \text { with } I\left(\beta^{\vee}, \alpha\right)=-1 \text { and } k(\beta)=2 k(\alpha),
\end{array}\right. \\
& \left\{\begin{array}{l}
{\left[E_{\alpha},\left[E_{\alpha},\left[E_{\alpha^{*}}, E_{\beta}\right]\right]\right]=0,\left[E_{\alpha},\left[E_{\alpha^{*}},\left[E_{\alpha^{*}}, E_{\beta}\right]\right]\right]=0,} \\
{\left[E_{-\alpha},\left[E_{-\alpha},\left[E_{-\alpha^{*}}, E_{-\beta}\right]\right]\right]=0,\left[E_{-\alpha},\left[E_{-\alpha^{*}},\left[E_{-\alpha^{*}}, E_{-\beta}\right]\right]\right]=0} \\
\text { for } \alpha, \beta \in \Pi_{\text {af }} \text { with } I\left(\beta^{\vee}, \alpha\right)=-1 \text { and } k(\beta)=3 k(\alpha),
\end{array}\right. \\
& \left\{\begin{array}{l}
{\left[E_{\alpha^{*}}, E_{\beta^{*}}\right]=0,\left[E_{-\alpha^{*}}, E_{-\beta^{*}}\right]=0} \\
\text { for } \alpha, \beta \in \Pi_{\mathrm{af}} \text { with } I(\alpha, \beta)=0
\end{array}\right.
\end{aligned}
$$

Here we state one of our main theorems:

Theorem 1.1. Let $\left(\mathcal{E}_{\mathrm{af}}^{\natural}, \Pi_{\mathrm{af}}=\left\{\alpha_{0}, \ldots, \alpha_{l}\right\}, k\right)$ be an r.m.e.-datum. Assume $l \geq 2$. Then there exists an isomorphism $\varphi: \mathfrak{g}^{\triangle}\left(\mathcal{E}_{\mathrm{af}}^{\natural}, \Pi_{\mathrm{af}}, k\right) \rightarrow$ $\mathfrak{g}\left(\mathcal{E}_{\mathrm{af}}^{\natural}, \Pi_{\mathrm{af}}, k\right)$ such that $\varphi\left(h_{\sigma}\right)=h_{\sigma}\left(\sigma \in \mathcal{E}_{\mathrm{af}}^{\natural}\right), \varphi\left(E_{ \pm \alpha}\right)=E_{ \pm \alpha}, \varphi\left(E_{\alpha^{*}}\right)=$ $2^{-1}\left[H_{\alpha^{\vee}}^{(1)}, E_{\alpha}\right], \varphi\left(E_{-\alpha^{*}}\right)=-2^{-1}\left[H_{\alpha \vee}^{(-1)}, E_{-\alpha}\right]\left(\alpha \in \Pi_{\mathrm{af}}\right)$. Moreover $\varphi\left(\widehat{H}_{ \pm \alpha}^{(i)}\right)$ 
$=H_{ \pm \alpha}^{(i)}\left(\alpha \in \Pi_{\mathrm{af}}\right.$ and $\left.i \in \mathbb{Z}\right)$, where

$$
\widehat{H}_{\alpha^{\vee}}^{(i)}:= \begin{cases}\left(-2^{-1}\right)^{i-1}\left(\operatorname{ad} E_{\alpha^{*}} \operatorname{ad} E_{-\alpha}\right)^{i-1}\left[E_{-\alpha}, E_{\alpha^{*}}\right] & \text { if } i>0, \\ h_{\alpha^{\vee}} & \text { if } i=0, \\ -\left(-2^{-1}\right)^{-i-1}\left(\operatorname{ad} E_{-\alpha^{*}} \operatorname{ad} E_{\alpha}\right)^{-i-1}\left[E_{\alpha}, E_{-\alpha^{*}}\right] & \text { if } i<0,\end{cases}
$$

and $\widehat{H}_{-\alpha^{\vee}}^{(i)}:=-\widehat{H}_{\alpha^{\vee}}^{(i)}$.

The proof shall be given after Proposition 1.1 is stated below.

Let $S$ be a subset of $\Pi_{\text {af }}$. Let $\mathfrak{g}^{(S)}$ be the Lie algebra defined by the generators $Z, h_{\sigma}\left(\sigma \in \mathcal{E}_{\text {af }}^{\natural}\right), E_{ \pm \alpha}(\alpha \in S), H_{ \pm \alpha \vee}^{(i)}(\alpha \in S, i \in \mathbb{Z})$ and the same defining relations as $(\mathrm{H} j)(1 \leq j \leq 8)$. Let $\mathfrak{g}^{(\triangle ; S)}$ be the Lie algebra defined by the generators $h_{\sigma}\left(\sigma \in \mathcal{E}_{\mathrm{af}}^{\natural}\right), E_{ \pm \alpha}, E_{ \pm \alpha^{*}}(\alpha \in S)$ and the same defining relations as $(\mathrm{S} j)(1 \leq j \leq 9)$.

Proposition 1.1. Keep the notation as above. Let $\alpha, \beta \in \Pi_{\mathrm{af}}$ be such that $\alpha \neq \beta$ and $I(\alpha, \beta) \neq 0$. Let $S:=\{\alpha, \beta\}$. Then there exists an isomorphism $\varphi^{(S)}: \mathfrak{g}^{(\triangle ; S)} \rightarrow \mathfrak{g}^{(S)}$ satisfying the same equalities as in Theorem 1.1 with $\varphi^{(S)}$ in place of $\varphi$.

The proposition follows immediately from Lemma 2.2 and Propositions 2.1 and 2.2 , which shall be given in $\S 2$.

Proof of Theorem 1.1. By Proposition 1.1, we see that for $\alpha, \beta \in \Pi_{\mathrm{af}}$ with $\alpha \neq \beta$ and $I(\alpha, \beta) \neq 0$,

$$
h_{a}, h_{\Lambda_{a}}, h_{\gamma^{\vee}}, E_{ \pm \gamma}, \widehat{H}_{\gamma^{\vee}}^{(i)}(\gamma \in\{\alpha, \beta\})
$$

satisfy the relations $(\mathrm{H} j), 1 \leq j \leq 8$, with $\widehat{H}_{\mu^{\vee}}^{(i)}$ in place of $H_{\mu^{\vee}}^{(i)}$.

From the definition of $\widehat{H}_{\mu^{\vee}}^{(i)}$, it is clear that for $\alpha, \beta \in \Pi_{\mathrm{af}}$ with $I(\alpha, \beta)=0$, the elements $E_{ \pm \gamma}, \widehat{H}_{\gamma^{\vee}}^{(i)}(\gamma=\alpha, \beta)$ satisfy the same relations as (H3), (H5), (H6) and (H8) with $\widehat{H}_{\gamma^{\vee}}^{(i)}$ in place of $H_{\gamma^{\vee}}^{(i)}$.

By the above argument, we see that there exists a homomorphism $\chi: \mathfrak{g} \rightarrow$ $\mathfrak{g}^{\triangle}$ such that $\chi\left(h_{\sigma}\right)=h_{\sigma}, \chi\left(E_{ \pm \alpha}\right)=E_{ \pm \alpha}, \chi\left(H_{ \pm \alpha^{\vee}}^{(i)}\right)=\widehat{H}_{ \pm \alpha^{\vee}}^{(i)}$.

Using again Proposition 1.1 and an argument similar to the above one, we can easily see that the homomorphism $\varphi: \mathfrak{g}^{\triangle} \rightarrow \mathfrak{g}$ in the statement can be defined. We can easily see that $\varphi$ is the inverse map of $\chi$. 


\section{§2. Rank Two Affine Lie Algebras}

\section{$\S 2.1$. Three kinds of definitions}

The automorphism $n_{\alpha}$. Let $\left(\mathcal{E}_{\mathrm{af}}^{\natural}, \Pi_{\mathrm{af}}, k\right)$ be an r.m.e.-datum. Let $\mathfrak{g}^{\triangle}=$ $\mathfrak{g}^{\triangle}\left(\mathcal{E}_{\mathrm{af}}^{\natural}, \Pi_{\mathrm{af}}, k\right)$. For $\alpha \in \Pi_{\mathrm{af}} \cup-\Pi_{\mathrm{af}}$, define the automorphism $n_{\alpha}: \mathfrak{g}^{\triangle} \rightarrow \mathfrak{g}^{\triangle}$ by

$$
n_{\alpha}:=\left(\exp \operatorname{ad} E_{\alpha}\right)\left(\exp \operatorname{ad} E_{-\alpha}\right)\left(\exp \operatorname{ad} E_{\alpha}\right) .
$$

It is well-known that

$$
n_{\alpha}\left(h_{\sigma}\right)=h_{s_{\alpha}(\sigma)},
$$

where $s_{\alpha}(\sigma)=\sigma-\frac{2 I(\alpha, \sigma)}{I(\alpha, \alpha)} \alpha$. Moreover we have:

$$
\begin{aligned}
& n_{\alpha} E_{\alpha}=-E_{-\alpha}, \quad n_{\alpha} h_{\alpha^{\vee}}=-h_{\alpha^{\vee}}, \quad n_{\alpha} E_{-\alpha}=-E_{\alpha}, \\
& \left\{\begin{array}{l}
n_{\alpha} E_{ \pm \alpha^{*}}=\frac{1}{2}\left(\operatorname{ad} E_{\mp \alpha}\right)^{2} E_{ \pm \alpha^{*}}, \\
n_{\alpha}\left[E_{\mp \alpha}, E_{ \pm \alpha^{*}}\right]=-\left[E_{\mp \alpha}, E_{ \pm \alpha^{*}}\right], \\
n_{\alpha}\left(\operatorname{ad} E_{\mp \alpha}\right)^{2} E_{ \pm \alpha^{*}}=2 E_{ \pm \alpha^{*}}
\end{array}\right.
\end{aligned}
$$

and, for $\beta, \alpha \in \Pi_{\mathrm{af}}$ with $\alpha \neq \beta$, we have:

$$
n_{\alpha}\left(\operatorname{ad} E_{ \pm \alpha}\right)^{i} E_{ \pm \mu}=\frac{(-1)^{i} i !}{(M-i) !}\left(\operatorname{ad} E_{ \pm \alpha}\right)^{M-i} E_{ \pm \mu}
$$

where $\mu:=\beta$ or $\beta^{*}, M:=-\frac{2 I(\alpha, \beta)}{I(\alpha, \alpha)}$ and $0 \leq i \leq M$.

The formulas in (2.1)-(2.4) follows immediately from Lemma 2.1 below.

Let $\mathfrak{a}$ be a Lie algebra and let $X_{i}(i \in \mathbf{I})$ be generators of $\mathfrak{a}$. We say that $X \in \mathfrak{a}$ is locally nilpotent if for every $i \in \mathbf{I},(\operatorname{ad} X)^{m_{i}} X_{i}=0$ for some $m_{i} \in \mathbb{N}$. For such an $X$, we can define $\exp (\operatorname{ad} X) \in \operatorname{Aut}(\mathfrak{a})$. Let $C=\left(X^{+}, Y, X^{-}\right)$be a triple consisting of elements $X^{+}, Y, X^{-}$of $\mathfrak{a}$. We say that such a $C$ is an $\mathrm{sl}_{2}$-triple if $\left[X^{+}, X^{-}\right]=-2 Y$ and $\left[Y, X^{ \pm}\right]= \pm 2 X^{ \pm}$. Here notice that $\left[X^{+}, X^{-}\right]$ is not $2 Y$ but $-2 Y$. If $X^{ \pm}$are locally nilpotent, we say that $C$ is locally finite and define

$$
n(C):=\exp \left(\operatorname{ad} X^{+}\right) \exp \left(\operatorname{ad} X^{-}\right) \exp \left(\operatorname{ad} X^{+}\right) \in \operatorname{Aut}(\mathfrak{a}) .
$$

Lemma 2.1. Keep the notation as above. Let $C=\left(X^{+}, Y, X^{-}\right)$be a locally finite $\operatorname{sl}_{2}$ triple. Let $n:=n(C)$. Let $Z \in \mathfrak{a}$ be such that $\left[X^{-}, Z\right]=0$ and $[Y, Z]=-m Z$ for some $m \in \mathbb{Z}_{+}$. Then $\left(\operatorname{ad} X^{+}\right)^{m+1} Z=0$ and

$$
n\left(\left(\operatorname{ad} X^{+}\right)^{i} Z\right)=\frac{(-1)^{i} i !}{(m-i) !}\left(\operatorname{ad} X^{+}\right)^{m-i} Z .
$$

for $0 \leq i \leq m$. 
Proof. Use a representation theory of $\mathrm{sl}_{2}$, especially the completely reducibility and the fact that

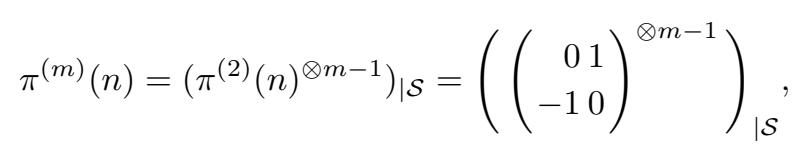

where $\pi^{(m)}$ is the $m$-dimensional irreducible representation $\pi^{(m)}$ of $\left\langle X^{+}, Y\right.$, $\left.X^{-}\right\rangle \cong \mathrm{sl}_{2}$, and $\mathcal{S}$ is the subspace of the symmetric tensors.

Kac-Moody Algebras. Let $\left(\mathcal{E}_{\mathrm{af}}, \Pi_{\mathrm{af}}\right)$ be an affine datum. Let $\mathbf{K M}=$ $\operatorname{KM}\left(\mathcal{E}_{\text {af }}, \Pi_{\text {af }}\right)$ be the Lie algebra defined by generators $h_{\sigma}\left(\sigma \in \mathcal{E}_{\text {af }}\right), E_{\alpha}$ $\left(\alpha \in \Pi_{\mathrm{af}} \cup-\Pi_{\mathrm{af}}\right)$ and relations:

$$
\begin{aligned}
x h_{\sigma}+y h_{\tau} & =h_{x \sigma+y \tau}, \quad\left[h_{\sigma}, h_{\tau}\right]=0, \\
{\left[h_{\sigma}, E_{\alpha}\right] } & =I(\sigma, \alpha) E_{\alpha}, \\
\left(\operatorname{ad} E_{\alpha}\right)^{1-J\left(\alpha^{\vee}, \beta\right)} E_{\beta} & = \begin{cases}h_{-\alpha} \vee & \text { if } \alpha+\beta=0, \\
0 & \text { otherwise, }\end{cases}
\end{aligned}
$$

Then $\mathbf{K M}$ is the affine Lie algebra of type $\Gamma_{\mathrm{af}}\left(\mathcal{E}_{\mathrm{af}}, \Pi_{\mathrm{af}}\right)$.

Let PriAff be the set of affine data $\left(\mathcal{E}_{\mathrm{af}}, \Pi_{\mathrm{af}}=\left\{\alpha_{0}, \alpha_{1}, \alpha_{2}\right\}\right)$ such that $\Gamma_{\mathrm{af}}\left(\mathcal{E}_{\mathrm{af}}, \Pi_{\mathrm{af}}\right)$ is $A_{2}^{(1)}, C_{2}^{(1)}, G_{2}^{(1)}, D_{3}^{(2)}$ or $D_{4}^{(3)}$. Notice that $A_{4}^{(2)} \notin$ PriAff.

Let $\left(\mathcal{E}_{\mathrm{af}}, \Pi_{\mathrm{af}}\right) \in$ PriAff. Define the function $g: \Pi_{\mathrm{af}, \mathrm{fi}} \cup-\Pi_{\mathrm{af}, \mathrm{fi}} \rightarrow\{1,2,3\}$ by:

$$
\left(g\left( \pm \alpha_{1}\right), g\left( \pm \alpha_{2}\right)\right)= \begin{cases}(1,1) & \text { if } \Gamma_{\mathrm{af}}\left(\mathcal{E}_{\mathrm{af}}, \Pi_{\mathrm{af}}\right)=A_{2}^{(1)}, C_{2}^{(1)} \text { or } G_{2}^{(1)} \\ (2,1) & \text { if } \Gamma_{\mathrm{af}}\left(\mathcal{E}_{\mathrm{af}}, \Pi_{\mathrm{af}}\right)=D_{3}^{(2)} \\ (1,3) & \text { if } \Gamma_{\mathrm{af}}\left(\mathcal{E}_{\mathrm{af}}, \Pi_{\mathrm{af}}\right)=D_{4}^{(3)}\end{cases}
$$

Let $\mathbf{G}=\mathbf{G}\left(\mathcal{E}_{\mathrm{af}}, \Pi_{\mathrm{af}}\right)$ be the Lie algebra defined by generators $h_{\sigma}\left(\sigma \in \mathcal{E}_{\mathrm{af}}\right), E_{\alpha}$ $\left(\alpha \in \Pi_{\mathrm{af}, \mathrm{fi}} \cup-\Pi_{\mathrm{af}, \mathrm{fi}}\right), H_{\alpha^{\vee}}^{(i)}\left(\alpha \in \Pi_{\mathrm{af}, \mathrm{fi}} \cup-\Pi_{\mathrm{af}, \mathrm{fi}}, i \in \mathbb{Z}\right)$ and relations:

$(\mathrm{PH} j)(1 \leq j \leq 8) \quad\left\{\begin{array}{l}\text { The relations obtained from those of }(\mathrm{H} j) \\ \text { by replacing } k \text { and } a \text { with } g \text { and }-\delta .\end{array}\right.$

Let $\mathbf{G}^{\triangle}=\mathbf{G}^{\triangle}\left(\mathcal{E}_{\mathrm{af}}, \Pi_{\mathrm{af}}\right)$ be the Lie algebra defined by generators $h_{\sigma}\left(\sigma \in \mathcal{E}_{\mathrm{af}}\right)$, $E_{\alpha}, E_{\alpha^{*}}\left(\alpha \in \Pi_{\mathrm{af}, \mathrm{fi}} \cup-\Pi_{\mathrm{af}, \mathrm{fi}}, i \in \mathbb{Z}\right)$ and relations:

$$
(\mathrm{PS} j)(1 \leq j \leq 9) \quad\left\{\begin{array}{l}
\text { The relations obtained from those of }(\mathrm{S} j) \\
\text { by replacing } k \text { and } a \text { with } g \text { and }-\delta .
\end{array}\right.
$$


It is easy to see:

Lemma 2.2. Let $\left(\mathcal{E}_{\mathrm{af}}^{\natural}, \Pi_{\mathrm{af}}, k\right)$ be an r.m.e.-datum. Assume $l \geq 2$. Let $\alpha, \beta \in \Pi_{\mathrm{af}}$ be such that $\alpha \neq \beta$ and $I(\alpha, \beta) \neq 0$. Let $S:=\{\alpha, \beta\}$. Then there exist $\left(\mathcal{E}_{\mathrm{af}}^{\prime}, \Pi_{\mathrm{af}}^{\prime}\right) \in$ PriAff, an injective isometric map $p^{(S)}: \mathcal{E}_{\mathrm{af}}^{\prime} \rightarrow \mathcal{E}_{\mathrm{af}}^{\natural}$ and $c \in\{1,2\}$ such that $p^{(S)}\left(\Pi_{\mathrm{af}, \mathrm{fi}}\right)=S, p^{(S)}(\delta)=-c a, p^{(S)}\left(\Lambda_{\delta}\right)=-c^{-1} \Lambda_{a}$, and $k\left(p\left(\alpha_{i}\right)\right)=c g\left(\alpha_{i}\right)$. (Notice that $\mathbf{G}=\mathbf{G}\left(\mathcal{E}_{\mathrm{af}}^{\prime}, \Pi_{\mathrm{af}}^{\prime}\right)$ can be identified with a subalgebra of $\mathfrak{g}^{(S)}$ by identifying $h_{\sigma}, E_{ \pm \alpha}, H_{\alpha^{\vee}}^{(i)}$ with $h_{p(\sigma)}, E_{ \pm p(\alpha)}, H_{p(\alpha)^{\vee}}^{(i)}$. Under the identification, we have $\mathfrak{g}^{(S)}=\mathbf{G} \oplus \mathfrak{r}$, where $\mathfrak{r}:=\left\{h_{\tau} \mid I\left(\tau, \mathcal{E}_{\text {af }}^{\prime}\right)=\{0\}\right\}$. Similar things hold for $\mathbf{G}^{\triangle}\left(\mathcal{E}_{\mathrm{af}}^{\prime}, \Pi_{\mathrm{af}}^{\prime}\right)$ and $\mathfrak{g}^{(\triangle ; S)}$.)

\section{$\S 2.2$. Isomorphisms between rank two affine algebras}

We use the same symbols for elements, $h_{\sigma}, E_{\alpha}$ etc., of the Lie algebras treated in this paper. In $\S 2.1$, we introduced the automorphism $n_{\alpha}$ of $\mathfrak{g}^{\triangle}$. We shall also denote by $n_{\alpha}$ the automorphism $n\left(\left(E_{\alpha}, h_{\alpha^{\vee}}, E_{-\alpha}\right)\right)$ even for the other Lie algebras. Moreover the same formulas as (2.1)-(2.4) also hold for the algebras. They shall also be referred to as (2.1)-(2.4). Let $\mathfrak{a}$ be a Lie algebra treated in this paper. Let $\mathcal{E}_{\mathfrak{a}}:=\mathcal{E}_{\text {af }}$ if $\mathfrak{a}=\mathbf{K M}, \mathbf{G}$ or $\mathbf{G}^{\triangle}$. Otherwise let $\mathcal{E}_{\mathfrak{a}}:=\mathcal{E}_{\mathrm{af}}^{\natural}$. Let $\mathfrak{h}_{\mathfrak{a}}$ be the subalgebra $\left\{h_{\sigma} \mid \sigma \in \mathcal{E}_{\mathfrak{a}}\right\}$ of $\mathfrak{a}$; by abuse of the notation, we shall also denote $\mathfrak{h}_{\mathfrak{a}}$ by $\mathfrak{h}$. We denote by $\mathfrak{a}_{\sigma}$ the weight space with respect to $\sigma \in \mathcal{E}$, i.e.,

$$
\mathfrak{a}_{\sigma}:=\left\{X \in \mathfrak{a} \mid\left[h_{\tau}, X\right]=I(\sigma, \tau) X\left(\tau \in \mathcal{E}_{\mathfrak{a}}\right)\right\} .
$$

The following lemma shall be used to prove Proposition 2.1.

Lemma 2.3. Let $\left(\mathcal{E}_{\mathrm{af}}, \Pi_{\mathrm{af}}\right) \in$ PriAff. For $\mathbf{G}=\mathbf{G}\left(\mathcal{E}_{\mathrm{af}}, \Pi_{\mathrm{af}}\right)$ and $\mathbf{K M}=$ $\mathbf{K M}\left(\mathcal{E}_{\mathrm{af}}, \Pi_{\mathrm{af}}\right)$, we have $\operatorname{dim} \mathbf{G}_{\sigma} \leq \operatorname{dim} \mathbf{K M}_{\sigma}$ for $\sigma \in \mathcal{E}_{\mathrm{af}}$.

Proof. We use the same argument as in [SY, §5]. Recall the elements $\alpha_{1}$, $\alpha_{2} \in \Pi_{\mathrm{af}, \mathrm{fi}}$. Let $Q_{0}:=\mathbb{Z} \delta, Q_{+}:=\left(\mathbb{Z}_{+} \alpha_{1}+\mathbb{Z}_{+} \alpha_{2}+Q_{0}\right) \backslash Q_{0}$ and $Q_{-}:=-Q_{+}$. Let $Q^{\text {re }}:=\cup_{i=1,2} \cup_{w \in W_{\mathrm{af}, \mathrm{fi}}}\left(w\left(\alpha_{i}\right)+\mathbb{Z} g\left(\alpha_{i}\right) \delta\right)$, where $W_{\mathrm{af}, \mathrm{fi}}=\left\langle s_{\alpha_{1}}, s_{\alpha_{2}}\right\rangle$. Notice that $Q^{\text {re }} \subset Q_{+} \cup Q_{-}$. Using the relations $(\mathrm{PH} j), 1 \leq j \leq 8$, we can easily see the facts below:

(1) $\mathbf{G}=\bigoplus_{\sigma \in\left(Q_{+} \cup Q_{0} \cup Q_{-}\right)} \mathbf{G}_{\sigma}$.

(2) $\mathbf{G}_{ \pm \alpha_{i}+g\left(\alpha_{i}\right) n \delta}=\mathbb{C}\left[H_{ \pm \alpha_{i}^{\vee}}^{(-n)}, E_{ \pm \alpha_{i}}\right]$ and $\mathbf{G}_{ \pm m \alpha_{i}+n \delta}=\{0\}$ for $m \geq 2$ or $n \notin g\left(\alpha_{i}\right) \mathbb{Z}$. 
(3) For $\sigma \in Q_{+} \backslash \cup_{i=1,2}\left(\mathbb{Z}_{+} \alpha_{i}+Q_{0}\right)$, we have $\mathbf{G}_{ \pm \sigma}=\sum\left[\mathbf{G}_{ \pm \sigma_{1}}, \mathbf{G}_{ \pm \sigma_{1}}\right]$, where the summation is taken over $\sigma_{1}, \sigma_{2} \in Q_{+}$with $\sigma_{1}+\sigma_{2}=\sigma$. $x \notin \mathbb{Z}$.

(4) $\mathbf{G}_{0}=\mathfrak{h}$ and $\mathbf{G}_{n \delta}=\sum_{i=1,2} \mathbb{C} H_{\alpha_{i}^{\vee}}^{\left(n / g\left(\alpha_{i}\right)\right)}$ for $n \neq 0$, where $H_{\alpha_{i}^{\vee}}^{(x)}:=0$ if

Moreover we notice the facts

(5) $\operatorname{dim} \mathbf{G}_{\sigma}=\operatorname{dim} n_{\alpha_{i}} \mathbf{G}_{\sigma}=\operatorname{dim} \mathbf{G}_{\beta}$, where $\beta=s_{\alpha_{i}}(\sigma)=\sigma-I\left(\alpha_{i}^{\vee}, \sigma\right) \alpha_{i}$.

(6) For each $\sigma \in Q_{+}$, since $I(\sigma, \sigma)>0$, we have $I\left(\alpha_{i}^{\vee}, \sigma\right)>0$ for some $i$.

By using the facts (1)-(6) and by using induction on $r_{1}+r_{2}$ for $\sigma=$ $r_{1} \alpha_{1}+r_{2} \alpha_{2}+n \delta \in Q_{+}$, we see:

$$
\begin{aligned}
\operatorname{dim} \mathbf{G}_{\sigma} \leq\left\{\begin{array}{l}
1 \text { if } \sigma \in Q^{\text {re }} \\
4 \text { if } \sigma=0, \\
2 \text { if } \sigma=n \delta \text { and } n / g\left(\alpha_{1}\right), n / g\left(\alpha_{2}\right) \in \mathbb{Z} \backslash\{0\}, \\
1 \text { if } \sigma=n \delta \text { and } n / g\left(\alpha_{1}\right) \notin \mathbb{Z} \text { or } n / g\left(\alpha_{1}\right) \notin \mathbb{Z}, \\
0 \text { otherwise }
\end{array}\right. \\
=\operatorname{dim} \mathbf{K M}_{\sigma},
\end{aligned}
$$

where the last equality can be seen by the concrete description of $\mathbf{K M}$ given in $[\mathrm{K}$, Chapters 7,8$]$.

Let $\left(\mathcal{E}_{\mathrm{af}}, \Pi_{\mathrm{af}}=\left\{\alpha_{0}, \alpha_{1}, \alpha_{2}\right\}\right) \in$ PriAff. Define the elements $\widetilde{E}_{\alpha^{*}}, \widetilde{E}_{-\alpha^{*}}$, $\widetilde{H}_{ \pm \alpha^{\vee}}^{(i)}\left(\alpha \in \Pi_{\mathrm{af}, \mathrm{fi}}, i \in \mathbb{Z}\right)$ of $\mathbf{K M}\left(\mathcal{E}_{\mathrm{af}}, \Pi_{\mathrm{af}}\right)$ as follows:

(1) If $\Gamma_{\mathrm{af}}\left(\mathcal{E}_{\mathrm{af}}, \Pi_{\mathrm{af}}\right)=A_{2}^{(1)}$, put $\widetilde{E}_{ \pm \alpha_{2}^{*}}:=n_{\alpha_{1}}^{-1} E_{\mp \alpha_{0}}$ and $\widetilde{E}_{ \pm \alpha_{1}^{*}}:=-\left[\left[E_{\mp \alpha_{2}}\right.\right.$, $\left.\left.\widetilde{E}_{ \pm \alpha_{2}^{*}}\right], E_{ \pm \alpha_{1}}\right]$.

(2) If $\Gamma_{\mathrm{af}}\left(\mathcal{E}_{\mathrm{af}}, \Pi_{\mathrm{af}}\right)=C_{2}^{(1)}$, put $\widetilde{E}_{ \pm \alpha_{2}^{*}}:=n_{\alpha_{1}}^{-1} E_{\mp \alpha_{0}}$ and $\widetilde{E}_{ \pm \alpha_{1}^{*}}:=-\left[\left[E_{\mp \alpha_{2}}\right.\right.$, $\left.\left.\widetilde{E}_{ \pm \alpha_{2}^{*}}\right], E_{ \pm \alpha_{1}}\right]$.

(3) If $\Gamma_{\mathrm{af}}\left(\mathcal{E}_{\mathrm{af}}, \Pi_{\mathrm{af}}\right)=G_{2}^{(1)}$, put $\widetilde{E}_{ \pm \alpha_{2}^{*}}:=n_{\alpha_{1}}^{-1} n_{\alpha_{2}}^{-1} E_{\mp \alpha_{0}}$ and $\widetilde{E}_{ \pm \alpha_{1}^{*}}:=$ $-\left[\left[E_{\mp \alpha_{2}}, \widetilde{E}_{ \pm \alpha_{2}^{*}}\right], E_{ \pm \alpha_{1}}\right]$.

(4) If $\Gamma_{\mathrm{af}}\left(\mathcal{E}_{\mathrm{af}}, \Pi_{\mathrm{af}}\right)=D_{3}^{(2)}$, put $\widetilde{E}_{ \pm \alpha_{2}^{*}}:=n_{\alpha_{1}}^{-1} E_{\mp \alpha_{0}}$ and $\widetilde{E}_{ \pm \alpha_{1}^{*}}:=4^{-1} \times$ $\left[\left[\left[\left[E_{\mp \alpha_{2}}, \widetilde{E}_{ \pm \alpha_{2}^{*}}\right], E_{\mp \alpha_{2}}\right], \widetilde{E}_{ \pm \alpha_{2}^{*}}\right], E_{ \pm \alpha_{1}}\right]$.

(5) If $\Gamma_{\mathrm{af}}\left(\mathcal{E}_{\mathrm{af}}, \Pi_{\mathrm{af}}\right)=D_{4}^{(3)}$, put $\widetilde{E}_{ \pm \alpha_{1}^{*}}:=n_{\alpha_{2}}^{-1} n_{\alpha_{1}}^{-1} E_{\mp \alpha_{0}}$ and $\widetilde{E}_{ \pm \alpha_{2}^{*}}:=$ $-12^{-1}\left[\left[\left[\left[\left[\left[E_{\mp \alpha_{1}}, \widetilde{E}_{ \pm \alpha_{1}^{*}}\right], E_{\mp \alpha_{1}}\right], \widetilde{E}_{ \pm \alpha_{1}^{*}}\right], E_{\mp \alpha_{1}}\right], \widetilde{E}_{ \pm \alpha_{1}^{*}}\right], E_{ \pm \alpha_{2}}\right]$.

(6) Define $\widetilde{H}_{ \pm \alpha^{\vee}}^{(i)}$ in the same way as that for $H_{ \pm \alpha^{\vee}}^{(i)}$ in (1.2) with $h_{\alpha^{\vee}}, E_{ \pm \alpha}$, $\widetilde{E}_{ \pm \alpha^{*}}$ in place of $h_{\alpha^{\vee}}, E_{ \pm \alpha}, E_{ \pm \alpha^{*}}$.

The two propositions below were used in the proof of Proposition 1.1.

Proposition 2.1. Let $\left(\mathcal{E}_{\mathrm{af}}, \Pi_{\mathrm{af}}\right) \in$ PriAff. Then there exists a unique isomorphism $\xi: \mathbf{G}\left(\mathcal{E}_{\mathrm{af}}, \Pi_{\mathrm{af}}\right) \rightarrow \mathbf{K M}\left(\mathcal{E}_{\mathrm{af}}, \Pi_{\mathrm{af}}\right)$ such that $\xi\left(h_{\sigma}\right)=h_{\sigma}, \xi\left(E_{ \pm \alpha}\right)=$ 
$E_{ \pm \alpha}$ and $\xi\left(H_{ \pm \alpha^{\vee}}^{(i)}\right)=\widetilde{H}_{ \pm \alpha^{\vee}}^{(i)}, \xi\left(2^{-1}\left[H_{ \pm \alpha^{\vee}}^{( \pm 1)}, E_{ \pm \alpha}\right]\right)=\widetilde{E}_{ \pm \alpha^{*}}$, where $\sigma \in \mathcal{E}_{\text {af }}, \alpha \in$ $\Pi_{\mathrm{af}, \text { fi }}$ and $i \in \mathbb{Z}$.

Proof. By the concrete description [K, Chapters 7, 8] of the affine Lie algebras, we see that the epimorphism $\xi$ in the statement exists. By Lemma 2.3, we see that $\operatorname{dim} \mathbf{G}_{\sigma}=\operatorname{dim} \mathbf{K} \mathbf{M}_{\sigma}$ for all $\sigma$, and that $\xi$ is injective.

Proposition 2.2. Let $\left(\mathcal{E}_{\mathrm{af}}, \Pi_{\mathrm{af}}\right) \in$ PriAff. Then there exists a unique isomorphism $\theta: \mathbf{K M}\left(\mathcal{E}_{\mathrm{af}}, \Pi_{\mathrm{af}}\right) \rightarrow \mathbf{G}^{\triangle}\left(\mathcal{E}_{\mathrm{af}}, \Pi_{\mathrm{af}}\right)$ such that $\theta\left(h_{\sigma}\right)=h_{\sigma}, \theta\left(E_{ \pm \alpha}\right)$ $=E_{ \pm \alpha}$ and $\theta\left(\widetilde{E}_{ \pm \alpha^{*}}\right)=E_{ \pm \alpha^{*}}$, where $\sigma \in \mathcal{E}_{\mathrm{af}}, \alpha \in \Pi_{\mathrm{af}, \mathrm{fi}}$. Moreover $\theta\left(\widetilde{H}_{ \pm \alpha^{\vee}}^{(i)}\right)=$ $\widehat{H}_{ \pm \alpha^{\vee}}^{(i)}$, where $\alpha \in \Pi_{\mathrm{af}, \mathrm{f}}, i \in \mathbb{Z}$ and where $\widehat{H}_{ \pm \alpha^{\vee}}^{(i)} \in \mathbf{G}^{\triangle}\left(\mathcal{E}_{\mathrm{af}}, \Pi_{\mathrm{af}}\right)$ are defined in the same way as in (1.2).

Proof. Define the elements $\widehat{E}_{ \pm \alpha_{0}}$ of $\mathbf{G}^{\triangle}\left(\mathcal{E}_{\mathrm{af}}, \Pi_{\mathrm{af}}\right)$ by

$$
\widehat{E}_{ \pm \alpha_{0}}:= \begin{cases}n_{\alpha_{1}} E_{\mp \alpha_{2}^{*}} & \text { if } \Gamma_{\mathrm{af}}\left(\mathcal{E}_{\mathrm{af}}, \Pi_{\mathrm{af}}\right) \text { is } A_{2}^{(1)}, C_{2}^{(1)} \text { or } D_{3}^{(2)}, \\ n_{\alpha_{2}} n_{\alpha_{1}} E_{\mp \alpha_{2}^{*}} & \text { if } \Gamma_{\mathrm{af}}\left(\mathcal{E}_{\mathrm{af}}, \Pi_{\mathrm{af}}\right) \text { is } G_{2}^{(1)} \\ n_{\alpha_{1}} n_{\alpha_{2}} E_{\mp \alpha_{1}^{*}} & \text { if } \Gamma_{\mathrm{af}}\left(\mathcal{E}_{\mathrm{af}}, \Pi_{\mathrm{af}}\right) \text { is } D_{4}^{(3)} .\end{cases}
$$

We first show that the elements $h_{\sigma}, \widehat{E}_{ \pm \alpha_{0}}, E_{ \pm \alpha_{1}}, E_{ \pm \alpha_{2}}$ satisfy the same relations as (AS1), (AS2) and (AS3) in $\S 2.1$ with $\widehat{E}_{ \pm \alpha_{0}}$ in place of $E_{ \pm \alpha_{0}}$. It is clear from (2.1) that $\left[\widehat{E}_{\alpha_{0}}, \widehat{E}_{-\alpha_{0}}\right]=h_{-\alpha_{0}^{\vee}}$. So we only need to show that

$$
\left(\operatorname{ad} \widehat{E}_{\alpha_{0}}\right)^{1-J\left(\alpha_{0}^{\vee}, \gamma\right)}\left(E_{\gamma}\right)=0
$$

and

$$
\left(\operatorname{ad} \widehat{E}_{-\alpha_{0}}\right)^{1-J\left(-\alpha_{0}^{\vee},-\gamma\right)}\left(E_{-\gamma}\right)=0,
$$

where $\gamma= \pm \alpha_{i}(i=1,2)$. We shall prove the equality $(2.5)$ for each $\left(\mathcal{E}_{\mathrm{af}}, \Pi_{\mathrm{af}}\right) \in$ PriAff in $\S 2.3$. The equality (2.6) can be proved similarly.

Define the homomorphism $\theta: \mathbf{K M}\left(\mathcal{E}_{\mathrm{af}}, \Pi_{\mathrm{af}}\right) \rightarrow \mathbf{G}^{\triangle}\left(\mathcal{E}_{\mathrm{af}}, \Pi_{\mathrm{af}}\right)$ by $\theta\left(h_{\sigma}\right)=$ $h_{\sigma}, \theta\left(E_{ \pm \alpha_{i}}\right)=E_{ \pm \alpha_{i}}(1 \leq i \leq 2)$ and $\theta\left(E_{ \pm \alpha_{0}}\right)=\widehat{E}_{ \pm \alpha_{0}}$. Put $i=2$ if $\Gamma_{\mathrm{af}}\left(\mathcal{E}_{\mathrm{af}}, \Pi_{\mathrm{af}}\right)$ is $D_{4}^{(3)}$; and put $i=1$ otherwise. It is clear that $\theta\left(\widetilde{E}_{ \pm \alpha_{i}^{*}}\right)=E_{ \pm \alpha_{i}^{*}}$. Let $j \in\{1,2\}$ be such that $j \neq i$. By (PS5), we see that

$$
E_{ \pm \alpha_{j}^{*}} \in \mathbb{C}\left(\operatorname{ad} E_{\mp \alpha_{i}}\right)^{k\left(\alpha_{j}\right) / k\left(\alpha_{i}\right)}\left(\operatorname{ad} E_{ \pm \alpha_{i}^{*}}\right)^{k\left(\alpha_{j}\right) / k\left(\alpha_{i}\right)} E_{ \pm \alpha_{j}} .
$$

Hence $\theta$ is surjective. By Proposition 3.2 in $\S 3$, we see that $\operatorname{ker} \theta \cap \mathfrak{h}=\{0\}$. By the abstract definition $[K, \S 1.3]$ of the Kac-Moody algebra, we see that $\operatorname{ker} \theta=\{0\}$. Hence $\theta$ is an isomorphism. By the concrete description $[\mathrm{K}$, Chapters 7,8$]$ of the affine Lie algebras, we see that $\theta\left(\widetilde{E}_{ \pm \alpha_{j}^{*}}\right)=E_{ \pm \alpha_{j}^{*}}$. It is clear that $\theta\left(\widetilde{H}_{ \pm \alpha^{\vee}}^{(i)}\right)=\widehat{H}_{ \pm \alpha^{\vee}}^{(i)}$. 


\section{§2.3. Proof of (2.5)}

Here, we shall use the notation for the elements of $\Pi_{\mathrm{af}}$ as follows: $\alpha$ and $\beta$ denote the two elements of $\Pi_{\mathrm{af}, \mathrm{fi}}=\left\{\alpha_{1}, \alpha_{2}\right\}$ and are assumed to be such that $I(\alpha, \alpha) \leq I(\beta, \beta) ; \beta_{\triangle}$ denotes $\alpha_{0}$ if $\Gamma_{\mathrm{af}}\left(\mathcal{E}_{\mathrm{af}}, \Pi_{\mathrm{af}}\right)$ is $A_{2}^{(1)}, C_{2}^{(1)}$ or $G_{2}^{(1)} ; \alpha_{\triangle}$ denotes $\alpha_{0}$ if $\Gamma_{\mathrm{af}}\left(\mathcal{E}_{\mathrm{af}}, \Pi_{\mathrm{af}}\right)$ is $D_{3}^{(2)}$ or $D_{4}^{(3)}$. We shall use the formulas (2.1)-(2.4) and the relations $(\mathrm{PSj})(1 \leq j \leq 9)$, and often omit telling which formula or relation to use in the cases where equalities are explicit.

The $A_{2}^{(1)}$ case. Notice that $\beta_{\triangle}=\delta-(\alpha+\beta)=s_{\alpha}\left(-\beta^{*}\right)$. Then we have the Dynkin diagram $A_{2}^{(1)}$ :

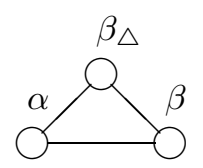

We have $\widehat{E}_{\beta_{\triangle}}=\left[E_{-\alpha}, E_{-\beta^{*}}\right]=\left[E_{-\alpha^{*}}, E_{-\beta}\right]$ and have:

$$
\left[E_{-\gamma}, \widehat{E}_{\beta_{\triangle}}\right]=0,\left(\operatorname{ad} E_{\gamma}\right)^{2} \widehat{E}_{\beta_{\triangle}}=0,
$$

where $\gamma$ is $\alpha$ or $\beta$. Notice that $\left[E_{\alpha}, \widehat{E}_{\beta_{\triangle}}\right]=-E_{-\beta^{*}}$. Then we have $\left(\operatorname{ad} \widehat{E}_{\beta_{\triangle}}\right)^{2} E_{\alpha}$ $=0$. Similarly we have $\left(\operatorname{ad} \widehat{E}_{\beta_{\triangle}}\right)^{2} E_{\beta}=0$.

The $C_{2}^{(1)}$ case. Notice that $\beta_{\triangle}=\delta-(2 \alpha+\beta)=s_{\alpha}\left(-\beta^{*}\right)$. We have the Dynkin diagram $C_{2}^{(1)}$ :

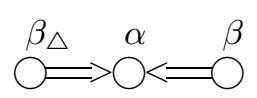

We have:

$$
\begin{array}{rll}
\widehat{E}_{\beta_{\triangle}} & =\frac{1}{2}\left[E_{-\alpha},\left[E_{-\alpha}, E_{-\beta^{*}}\right]\right] & (\text { by }(2.4)) \\
& =\frac{1}{2}\left[E_{-\alpha^{*}},\left[E_{-\alpha}, E_{-\beta}\right]\right] & (\text { by }(\mathrm{PS} 3) \text { and (PS5)). }
\end{array}
$$

It is clear that $\left[E_{-\alpha}, \widehat{E}_{\beta_{\Delta}}\right]=0,\left(\operatorname{ad} E_{\alpha}\right)^{3} \widehat{E}_{\beta_{\Delta}}=0$ and $\left[\widehat{E}_{\beta_{\Delta}}, E_{\beta}\right]=0$. We see that

$$
\left[\widehat{E}_{\beta_{\triangle}}, E_{-\beta}\right]=\frac{1}{2}\left[\left[E_{-\alpha^{*}}, E_{-\beta}\right],\left[E_{-\alpha}, E_{-\beta}\right]\right]=\frac{1}{4}\left(\operatorname{ad} E_{-\beta}\right)^{2}\left[E_{-\alpha^{*}}, E_{-\alpha}\right]=0 .
$$

We see that $\left(\operatorname{ad} \widehat{E}_{\beta_{\triangle}}\right)^{2} E_{\alpha}=n_{\alpha}\left(\operatorname{ad} E_{-\beta^{*}}\right)^{2}\left(-E_{-\alpha}\right)=0$.

The $G_{2}^{(1)}$ case. Notice that $\beta_{\triangle}:=\delta-(3 \alpha+2 \beta)=s_{\beta} s_{\alpha}\left(-\beta^{*}\right)$. Then we have the Dynkin diagram $G_{2}^{(1)}$ :

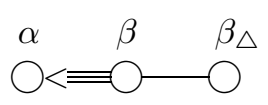


We have

$$
\begin{aligned}
\widehat{E}_{\beta_{\triangle}} & =n_{\beta} n_{\alpha} E_{-\beta^{*}}=n_{\beta}\left(\frac{1}{6}\left(\operatorname{ad} E_{-\alpha}\right)^{3} E_{-\beta^{*}}\right) \\
& =-\frac{1}{6}\left(\operatorname{ad} n_{\beta} E_{-\alpha}\right)^{2} n_{\beta}\left[E_{-\beta}, E_{-\alpha^{*}}\right]=\frac{1}{6}\left(\operatorname{ad}\left[E_{-\beta}, E_{-\alpha}\right]\right)^{2} E_{-\alpha^{*}}
\end{aligned}
$$

It is clear that:

$$
\left(\operatorname{ad} E_{\beta}\right)^{2} \widehat{E}_{\beta_{\triangle}}=\frac{1}{3}\left(\operatorname{ad} E_{-\alpha}\right)^{2} E_{-\alpha^{*}}=0
$$

Notice that:

$$
\left[\left[E_{-\alpha}, E_{-\beta}\right],\left[E_{-\alpha}, E_{-\beta^{*}}\right]\right]=\left[\left[E_{-\alpha}, E_{-\beta}\right],\left[E_{-\alpha^{*}}, E_{-\beta}\right]\right]=n_{\beta}\left[E_{-\alpha}, E_{-\alpha^{*}}\right]=0,
$$

and we see:

$$
\begin{aligned}
{\left[E_{-\beta}, \widehat{E}_{\beta_{\triangle}}\right] } & =\frac{1}{6}\left[\left[E_{-\beta}, E_{-\alpha}\right],\left[\left[E_{-\beta}, E_{-\alpha}\right],\left[E_{-\beta}, E_{-\alpha^{*}}\right]\right]\right]=0 \\
{\left[E_{-\alpha}, \widehat{E}_{\beta_{\triangle}}\right] } & =n_{\beta} n_{\alpha}\left[\frac{1}{2}\left[\left[E_{-\alpha},\left[E_{-\alpha}, E_{-\beta}\right]\right], E_{-\beta^{*}}\right]\right] \\
& =-\frac{1}{2} n_{\beta} n_{\alpha}\left[\left[E_{-\alpha} E_{-\beta}\right],\left[E_{-\alpha}, E_{-\beta^{*}}\right]\right]=0 \\
{\left[\widehat{E}_{\beta_{\triangle}}, E_{\alpha}\right] } & =\frac{1}{6}\left[\left[\left[E_{-\beta}, E_{-\alpha}\right],\left[\left[E_{-\beta^{*}}, E_{-\alpha}\right], E_{-\alpha}\right]\right], E_{\alpha}\right] \\
& =\frac{1}{6}\left(-3\left[E_{-\beta},\left[\left[E_{-\beta^{*}}, E_{-\alpha}\right], E_{-\alpha}\right]\right]-4\left[\left[E_{-\beta}, E_{-\alpha}\right],\left[E_{-\beta^{*}}, E_{-\alpha}\right]\right]\right) \\
& =\frac{1}{6}\left[\left[E_{-\beta^{*}}, E_{-\alpha}\right],\left[E_{-\beta}, E_{-\alpha}\right]\right]=0
\end{aligned}
$$

and

$$
\begin{aligned}
\left(\operatorname{ad} \widehat{E}_{\beta_{\triangle}}\right)^{2} E_{\beta} & =n_{\beta} n_{\alpha}\left(\operatorname{ad} E_{-\beta^{*}}\right)^{2}\left(\frac{1}{6}\left(\operatorname{ad} E_{-\alpha}\right)^{3} E_{-\beta}\right) \quad(\text { by }(2.4)) \\
& =-\frac{1}{3} n_{\beta} n_{\alpha}\left(\operatorname{ad}\left[E_{-\beta^{*}}, E_{-\alpha}\right]\right)^{2}\left[E_{-\beta}, E_{-\alpha}\right] \quad(\text { by }(\text { PS6 })) \\
& =0 .
\end{aligned}
$$

The $D_{3}^{(2)}$ case. Notice that $\alpha_{\triangle}=\delta-(\alpha+\beta)=s_{\beta}\left(-\alpha^{*}\right)$. We have the Dynkin diagram $D_{3}^{(2)}$ :

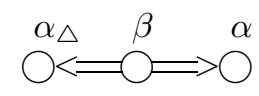


We see that $\widehat{E}_{\alpha_{\Delta}}=\left[E_{-\beta}, E_{-\alpha^{*}}\right]$. It is clear that $\left[E_{-\beta}, \widehat{E}_{\alpha_{\Delta}}\right]=0$ and $\left(\operatorname{ad} E_{\beta}\right)^{2} \widehat{E}_{\alpha_{\Delta}}=0$. It follows from (PS7) that $\left[E_{-\alpha}, \widehat{E}_{\alpha_{\Delta}}\right]=0$ and $\left(\operatorname{ad} \widehat{E}_{\alpha_{\Delta}}\right)^{3} E_{\beta}$ $=n_{\beta}\left(\operatorname{ad} E_{-\alpha^{*}}\right)^{3}\left(-E_{-\beta}\right)=0$. We have:

$$
\begin{aligned}
& {\left[\widehat{E}_{\alpha_{\Delta}}, E_{\alpha}\right]} \\
& =\left[\left[E_{-\beta}, E_{-\alpha^{*}}\right], E_{\alpha}\right] \\
& =\left[E_{-\beta},\left[E_{-\alpha^{*}}, E_{\alpha}\right]\right] \\
& =n_{\alpha}^{-1}\left[\frac{1}{2}\left[E_{-\alpha},\left[E_{-\alpha}, E_{-\beta}\right]\right],-\left[E_{-\alpha^{*}}, E_{\alpha}\right]\right] \\
& =n_{\alpha}^{-1}\left[\left[E_{-\alpha}, E_{-\beta}\right], E_{-\alpha^{*}}\right] \quad(\text { by }(\mathrm{PS} 2),(\mathrm{PS} 3) \text { and }(\mathrm{PS} 7)) \\
& =0 \quad(\text { by }(\mathrm{PS} 7)) .
\end{aligned}
$$

The $D_{4}^{(3)}$ case. Notice that $\alpha_{\triangle}=\delta-(2 \alpha+\beta)=s_{\alpha} s_{\beta}\left(-\alpha^{*}\right)$. The following is the Dynkin diagram $D_{4}^{(3)}$ :

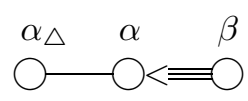

We have

$$
\begin{aligned}
\widehat{E}_{\alpha_{\triangle}} & =n_{\alpha} n_{\beta} E_{-\alpha^{*}}=n_{\alpha}\left[E_{-\beta}, E_{-\alpha^{*}}\right] \\
& =\left[\frac{1}{6}\left(\operatorname{ad} E_{-\alpha}\right)^{3} E_{-\beta}, \frac{1}{2}\left(\operatorname{ad} E_{\alpha}\right)^{2} E_{-\alpha^{*}}\right] \\
& =\frac{3}{12}\left(\left[\left(\operatorname{ad} E_{-\alpha}\right)^{2} E_{-\beta},\left[E_{\alpha}, E_{-\alpha^{*}}\right]\right]+\left[E_{\alpha},\left[\left(\operatorname{ad} E_{-\alpha}\right)^{2} E_{-\beta}, E_{-\alpha^{*}}\right]\right]\right) \\
& =\frac{1}{4}\left[\left(\operatorname{ad} E_{-\alpha}\right)^{2} E_{-\beta},\left[E_{\alpha}, E_{-\alpha^{*}}\right]\right] \quad(\text { by }(\mathrm{PS} 8)) \\
& =\left[\left[E_{-\alpha}, E_{-\beta}\right], E_{-\alpha^{*}}\right] .
\end{aligned}
$$

It is clear that $\left[\widehat{E}_{\alpha_{\Delta}}, E_{\beta}\right]=0$ and $\left[\widehat{E}_{\alpha_{\Delta}}, E_{-\alpha}\right]=0$. It follows that $\left[\widehat{E}_{\alpha_{\Delta}}, E_{-\beta}\right]=$ $n_{\beta}\left[E_{-\alpha}, E_{-\alpha^{*}}\right]=0$. We see that $\left(\operatorname{ad} E_{\alpha}\right)^{2} \widehat{E}_{\alpha_{\Delta}}=n_{\alpha}\left(\operatorname{ad} E_{-\alpha}\right)^{2}\left(n_{\beta} E_{-\alpha^{*}}\right)=$ $n_{\alpha}\left(\operatorname{ad} E_{-\alpha}\right)^{2}\left(\left[E_{-\beta} E_{-\alpha^{*}}\right]\right)=0$. We also see that:

$$
\begin{aligned}
\operatorname{ad} & \left(\widehat{E}_{\alpha_{\triangle}}\right)^{2} E_{\alpha} \\
& =-n_{\alpha}\left[\left[E_{-\beta}, E_{-\alpha^{*}}\right],\left[\left[E_{-\beta}, E_{-\alpha^{*}}\right], E_{-\alpha}\right]\right] \\
& \left.=n_{\alpha}\left[E_{-\alpha^{*}},\left[E_{-\beta},\left[\left[E_{-\beta}, E_{-\alpha^{*}}\right], E_{-\alpha}\right]\right]\right]\right] \\
& =n_{\alpha}\left[E_{-\alpha^{*}},\left[\left[E_{-\beta}, E_{-\alpha^{*}}\right],\left[E_{-\beta}, E_{-\alpha}\right]\right]\right] \\
& =n_{\alpha}\left[E_{-\alpha^{*}}, \frac{1}{2}\left(\operatorname{ad} E_{-\beta}\right)^{2}\left[E_{-\alpha^{*}}, E_{-\alpha}\right]\right] \\
& =0 .
\end{aligned}
$$




\section{§3. Root Spaces}

Let $\left(\mathcal{E}_{\mathrm{af}}=\left\{\alpha_{0}, \ldots, \alpha_{l}\right\}, k\right)$ be an affine datum. Recall the affine Lie algebra $\mathbf{K M}=\mathbf{K M}\left(\mathcal{E}_{\mathrm{af}}, \Pi_{\mathrm{af}}\right)$ in $\S 2.1$. Let $(\mid): \mathbf{K M} \times \mathbf{K M} \rightarrow \mathbb{C}$ be the same invariant form as in $\left[\mathrm{K}\right.$, Theorem 2.2]. Let $\mathbb{C}\left[t, t^{-1}\right]$ be the Laurent polynomial algebra. Define the Lie algebra structure on the vector space

$$
L(\mathbf{K M}, 1):=\mathbf{K M} \otimes \mathbb{C}\left[t, t^{-1}\right] \oplus \mathbb{C} v \oplus \mathbb{C} w
$$

by

$$
\begin{aligned}
& {\left[X_{1} \otimes a_{1}(t)+b_{1} v+c_{1} w, X_{2} \otimes a_{2}(t)+b_{2} v+c_{2} w\right]} \\
& =\left[X_{1}, X_{2}\right] \otimes a_{1}(t) a_{2}(t)+\left(X_{1} \mid X_{2}\right) \operatorname{Res}\left(\frac{\mathrm{d} a_{1}(t)}{\mathrm{d} t} a_{2}(t)\right) v \\
& \quad-c_{2} X_{1} \otimes t \frac{\mathrm{d} a_{1}(t)}{\mathrm{d} t}+c_{1} X_{2} \otimes t \frac{\mathrm{d} a_{2}(t)}{\mathrm{d} t}
\end{aligned}
$$

where $\operatorname{Res}\left(\sum x_{i} t^{i}\right)=x_{-1}$.

Let $\left(\mathcal{E}_{\mathrm{af}}^{\natural}, \Pi_{\mathrm{af}}=\left\{\alpha_{0}, \ldots, \alpha_{l}\right\}, k\right)$ be an r.m.e.-datum such that $l \geq 2$. Let $k^{\vee}(\alpha):=k(\alpha)^{-1} \max \left\{k(\alpha) \mid \alpha \in \Pi_{\mathrm{af}}\right\}$. Let $\mathcal{E}_{\mathrm{af}}^{b}$ be the $1+\sum k^{\vee}\left(\alpha_{i}\right)$ dimensional $\mathbb{C}$ vector space such that

(1) there exists a non-degenerate symmetric form $I: \mathcal{E}_{\mathrm{af}}^{b} \times \mathcal{E}_{\mathrm{af}}^{b} \rightarrow \mathbb{C}$,

(2) there exist linearly independent elements $\alpha_{i, j} \in \mathcal{E}_{\mathrm{af}}^{b}(0 \leq i \leq l, 1 \leq j \leq$ $\left.k^{\vee}\left(\alpha_{i}\right)\right)$ such that

$(2-1) I\left(\alpha_{i, j_{1}}, \alpha_{i, j_{2}}\right)=0$ if $j_{1} \neq j_{2}$,

(2-2) $I\left(\alpha_{i, j}, \alpha_{i, j}\right)=k^{\vee}\left(\alpha_{i}\right) I\left(\alpha_{i}, \alpha_{i}\right)$,

(2-3) $I\left(\alpha_{i_{1}, j_{1}}, \alpha_{i_{2}, j_{2}}\right)=0$ if $I\left(\alpha_{i_{1}}, \alpha_{i_{2}}\right)=0$,

(2-4) $I\left(\left(\alpha_{i_{1}, j_{1}}\right)^{\vee}, \alpha_{i_{2}, j_{2}}\right)=\delta_{j_{1}, j_{2}} I\left(\left(\alpha_{i_{1}}\right)^{\vee}, \alpha_{i_{2}}\right)$ if $I\left(\alpha_{i_{1}}, \alpha_{i_{2}}\right) \neq 0$

and $k\left(\alpha_{i_{1}}\right)=k\left(\alpha_{i_{2}}\right)$,

$(2-5)$ if $I\left(\alpha_{i_{1}}, \alpha_{i_{2}}\right) \neq 0$ and $k^{\vee}\left(\alpha_{i_{1}}\right)<k^{\vee}\left(\alpha_{i_{2}}\right)$, then

$$
\begin{aligned}
& I\left(\left(\alpha_{i_{1}, j_{1}}\right)^{\vee}, \alpha_{i_{2}, j_{2}}\right)=I\left(\left(\alpha_{i_{2}, j_{2}}\right)^{\vee}, \alpha_{i_{1}, j_{1}}\right) \\
& \quad=\left\{\begin{array}{l}
-1 \text { if } k^{\vee}\left(\alpha_{i_{1}}\right)=1, \\
-1 \text { if } k^{\vee}\left(\alpha_{i_{1}}\right)=2, k^{\vee}\left(\alpha_{i_{2}}\right)=4 \text { and } j_{1}-j_{2} \in 2 \mathbb{Z}, \\
0 \quad \text { otherwise. }
\end{array}\right.
\end{aligned}
$$

Let $\Pi_{\mathrm{af}}^{b}:=\left\{\alpha_{i, j}\right\}$. We see that $\left(\mathcal{E}_{\mathrm{af}}^{b}, \Pi_{\mathrm{af}}^{b}\right)$ is an affine datum. Let $\mathbf{K M}^{b}:=$ $\operatorname{KM}\left(\mathcal{E}_{\text {af }}^{b}, \Pi_{\text {af }}^{b}\right)$. 
Being inspired by $[\mathrm{P}]$, we have:

Proposition 3.1. Keep the notation as above. Then there exists a homomorphism:

$$
\chi: \mathfrak{g}^{\triangle}\left(\mathcal{E}_{\mathrm{af}}^{\natural}, \Pi_{\mathrm{af}}, k\right) \rightarrow L\left(\mathbf{K M}^{\mathrm{b}}, 1\right)
$$

such that

$$
\begin{gathered}
\chi\left(E_{ \pm \alpha_{i}}\right)=\sum_{j=1}^{k^{\vee}\left(\alpha_{i}\right)} E_{ \pm \alpha_{i, j}} \otimes 1 \\
\chi\left(E_{ \pm \alpha_{i}^{*}}\right)=\sum_{j=1}^{k^{\vee}\left(\alpha_{i}\right)} \exp \left( \pm \frac{\pi \sqrt{-1}}{k^{\vee}\left(\alpha_{i}\right)}\left(2 j-1-k^{\vee}\left(\alpha_{i}\right)\right)\right) E_{ \pm \alpha_{i, j}} \otimes t^{ \pm k\left(\alpha_{i}\right)}
\end{gathered}
$$

and $\chi\left(h_{a}\right)=v, \chi\left(h_{\Lambda_{a}}\right)=w$, and such that $\chi\left(h_{\sigma}\right) \neq 0$ for $\sigma \in \mathcal{E}_{\text {af }}^{\natural} \backslash\{0\}$.

By the same argument as above with $\mathbf{G}^{\triangle}$ in place of $\mathfrak{g}^{\triangle}$, we also have:

Proposition 3.2. $\quad$ Keep the notation as in the proof of Proposition 2.2. Then $\operatorname{ker} \theta \cap \mathfrak{h}=\{0\}$.

Root spaces of $\mathfrak{g}^{\triangle}$. Let $\mathfrak{g}^{\triangle}=\mathfrak{g}^{\triangle}\left(\mathcal{E}_{\mathrm{af}}^{\natural}, \Pi_{\mathrm{af}}, k\right)$ be as above. Recall the element $\widehat{H}_{\alpha^{\vee}}^{(i)}$ of $\mathfrak{g}^{\triangle}$ from Theorem 1.1, where $\alpha \in \Pi_{\mathrm{af}}$ and $i \in \mathbb{Z}$. Define the subset $R$ of $\mathcal{E}_{\text {af }}^{\natural}$ by

$$
R:=\bigcup_{w \in W} \bigcup_{\alpha \in \Pi_{\mathrm{af}}}(w(\alpha)+\mathbb{Z} k(\alpha) a),
$$

where $W$ is the affine Weyl group $\left\langle s_{\alpha} \mid \alpha \in \Pi_{\text {af }}\right\rangle$ (see also (0.1)). By Proposition 3.1 , we see that

$$
\left[\widehat{H}_{\alpha^{\vee}}^{(i)}, E_{\alpha}\right] \neq 0
$$

$\left(\alpha \in \Pi_{\mathrm{af}} \cup-\Pi_{\mathrm{af}}\right)$ since $\left[\left[\widehat{H}_{\alpha^{\vee}}^{(i)}, E_{\alpha}\right],\left[\widehat{H}_{\alpha^{\vee}}^{(-i)}, E_{-\alpha}\right]\right]=\frac{8}{I(\alpha, \alpha)} h_{\alpha+i k(\alpha) a} \neq 0$. By Theorem 1.1, Proposition 3.1 and (3.2) and by using the same argument as in the proof of Lemma 2.3 (see also [SY, §5]), we have:

Proposition 3.3 (See also $[\mathrm{Y}]$ ). $\quad$ As a $\mathbb{C}$-vector space,

$$
\mathfrak{g}^{\triangle}=\left(\bigoplus_{\beta \in R} \mathfrak{g}_{\beta}^{\triangle}\right) \bigoplus\left(\bigoplus_{m, n \in \mathbb{Z}} \mathfrak{g}_{m \delta+n a}^{\triangle}\right) .
$$


It also follows that:

$$
\operatorname{dim} \mathfrak{g}_{\beta}^{\triangle}=1
$$

for $\beta \in R$, and

$$
\mathfrak{g}_{0}^{\triangle}=\mathfrak{h}
$$

In particular, $\operatorname{dim} \mathfrak{g}_{0}^{\triangle}=l+4$. Moreover, for $\beta \in R$, there exist $\alpha \in \Pi_{\mathrm{af}}, i \in \mathbb{Z}$ and $\alpha_{i_{1}}, \ldots, \alpha_{i_{u}} \in \Pi_{\mathrm{af}}$ such that

$$
\mathfrak{g}_{\beta}^{\triangle}=\mathbb{C} n_{\alpha_{i_{1}}} \cdots n_{\alpha_{i_{u}}}\left[\widehat{H}_{\alpha^{\vee}}^{(i)}, E_{\alpha}\right] .
$$

We introduce the Lie algebra $\mathfrak{g}^{\natural}$, which was mentioned in Introduction.

Definition 3.1 (See also Introduction). Keep the notation as above. Assume $l \geq 2$. Let $\mathcal{B}:=\left\{\alpha,-\alpha, \alpha^{*},-\alpha^{*} \mid \alpha \in \Pi_{\mathrm{af}}\right\}, x_{\mu, \nu}:=\min \{n \in$ $\mathbb{N} \mid n \mu+\nu \notin R \cup \mathbb{C} a\}$ and $\mathcal{A}:=\left\{(\alpha, \beta, y) \in \Pi_{\mathrm{af}} \times \Pi_{\mathrm{af}} \times \mathbb{N} \mid \alpha \neq \beta, I(\alpha, \beta) \neq\right.$ $0, k(\alpha) y=k(\beta)\}$. Define the Lie algebra $\mathfrak{g}^{\natural}$ with generators:

$$
h_{\sigma}(\sigma \in \mathcal{E}), E_{\mu}(\mu \in \mathcal{B})
$$

and defining relations:

$$
\begin{cases}x h_{\sigma}+y h_{\tau}=h_{x \sigma+y \tau} & \text { for } x, y \in \mathbb{C} \text { and } \sigma, \tau \in \mathcal{E} \\ {\left[h_{\sigma}, h_{\tau}\right]=0} & \text { for } \sigma, \tau \in \mathcal{E} \\ {\left[h_{\sigma}, E_{\mu}\right]=I(\sigma, \mu) E_{\mu}} & \text { for } \sigma \in \mathcal{E} \text { and } \mu \in \mathcal{B} \\ {\left[E_{\mu}, E_{-\mu}\right]=h_{\mu^{\vee}}} & \text { for } \mu \in \mathcal{B}, \\ \left(\operatorname{ad} E_{\mu}\right)^{x_{\mu, \nu}} E_{\nu}=0 & \text { for } \mu, \nu \in \mathcal{B} \text { with } \mu+\nu \neq 0 \\ \left(\operatorname{ad} E_{ \pm \alpha^{*}}\right)^{y} E_{ \pm \beta}=\left(\operatorname{ad} E_{ \pm \alpha}\right)^{y} E_{ \pm \beta^{*}} & \text { for }(\alpha, \beta, y) \in \mathcal{A}, \\ \left(\operatorname{ad} E_{ \pm \alpha}\right)^{i}\left(\operatorname{ad} E_{ \pm \alpha^{*}}\right)^{y-i} E_{ \pm \beta}=0 & \text { for }(\alpha, \beta, y) \in \mathcal{A} \text { and } 1 \leq i \leq y-1\end{cases}
$$

The following theorem was also mentioned in Introduction.

Theorem 3.1. Assume $l \geq 2$. There exists an isomorphism $\psi: \mathfrak{g}^{\triangle} \rightarrow$ $\mathfrak{g}^{\natural}$ such that $\psi\left(h_{\sigma}\right)=h_{\sigma}$ and $\psi\left(E_{\mu}\right)=\varepsilon_{\mu} E_{\mu}$ for some $\varepsilon_{\mu}= \pm 1$.

Proof. The existence of the homomorphism $\psi$ is clear. By Proposition 3.3, we see that the inverse map $\psi^{-1}$ exists. 
Invariant form and a maximality theorem. Keep the notation as above. We shall also denote by (| ) the invariant form on $\mathbf{K M}^{\mathrm{b}}$ defined by:

$$
\begin{aligned}
& \left(X_{1} \otimes a_{1}(t)+b_{1} v+c_{1} w \mid X_{2} \otimes a_{2}(t)+b_{2} v+c_{2} w\right) \\
& =\left(X_{1} \mid X_{2}\right) \operatorname{Res}\left(t a_{1}(t) a_{2}(t)\right)+b_{1} c_{2}+b_{2} c_{1} .
\end{aligned}
$$

We shall also denote by $(\mid)$ the invariant form on $\mathfrak{g}^{\natural}$ defined by the composition of the above $(\mid)$ and $\chi \psi^{-1} \times \chi \psi^{-1}$. Then we have:

$$
\left(h_{\sigma} \mid h_{\tau}\right)=I(\sigma, \tau) \quad\left(\sigma, \tau \in \mathcal{E}_{\mathrm{af}}^{\natural}\right)
$$

and

$$
\operatorname{ker}(\mid) \subset \bigoplus_{\substack{m, n \in \mathbb{Z} \\(m, n) \neq(0,0)}} \mathfrak{g}_{m \delta+n a}^{\natural} .
$$

Theorem 3.2. Assume that $l \geq 2$ and $\Gamma_{\mathrm{af}}\left(\mathcal{E}_{\mathrm{af}}, \Pi_{\mathrm{af}}\right)$ is not $A_{l}^{(1)}$ (see $\S 1$ and notice $\Gamma(R, G) \neq A_{l}^{(1,1)}$ (see $\S 4$ and Appendix)). Let $\mathfrak{g}^{\sharp}=\mathfrak{g}^{\sharp}\left(\mathcal{E}_{\mathrm{af}}^{\natural}, \Pi_{\mathrm{af}}, k\right)$ be a Lie algebra satisfying:

(1) $\mathfrak{g}^{\sharp}$ has the same properties as (3.3)-(3.5) with $\mathfrak{g}^{\sharp}$ in place of $\mathfrak{g}^{\natural}$,

(2) $\mathfrak{g}^{\sharp}$ is generated by $\mathfrak{h}$ and $\mathfrak{g}_{\mu}^{\sharp}$ with $\mu \in \mathcal{B}$,

(3) There exists an invariant form on $\mathfrak{g}^{\sharp}$ having the same properties as in $(3.7)-(3.8)$.

Then there exists an epimorphism $\eta: \mathfrak{g}^{\natural} \rightarrow \mathfrak{g}^{\sharp}$ such that $\eta\left(h_{\sigma}\right)=h_{\sigma}$ for $\sigma \in \mathcal{E}_{\mathrm{af}}^{\natural}$.

Proof. By a well-known argument (see [K, Theorem 2.2]), we can choose basis elements $E_{\mu}$ of $\mathfrak{g}_{\mu}^{\sharp}$ with $\mu \in \mathcal{B}$ so that $\left[E_{\mu}, E_{-\mu}\right]=h_{\mu}$. Then $E_{\mu}$ 's satisfy the same equalities as (3.6) except for the sixth one. Notice $n_{\mu}=$ $n\left(\left(E_{\mu}, h_{\mu^{\vee}},-E_{-\mu}\right)\right) \in \operatorname{Aut}\left(\mathfrak{g}^{\sharp}\right)$ can be defined for $\mu \in \mathcal{B}$ (see $\left.\S 2.2\right)$. Assume that $(\alpha, \beta, y) \in \mathcal{A}$ (see (3.6)). Assume that if $y=1$, then $I\left(\alpha^{\vee}, \beta\right)=-1$. Then we have:

$$
n_{\alpha^{*}} E_{ \pm \beta}=c_{\alpha, \beta}^{ \pm 1} n_{\alpha} E_{ \pm \beta^{*}}
$$

for some $c_{\alpha, \beta} \in \mathbb{C} \backslash\{0\}$. We can normalize $E_{ \pm \gamma^{*}}$ so that $c_{\alpha, \beta}=1$ hold for all $\alpha, \beta$. Then the equality (3.9) is nothing but the sixth one. This completes the proof. 


\section{§4. Relations with Elliptic Root Basis}

Here we recall the notion of the elliptic diagram $[\mathrm{S},(5,2)]$. Let $\left(\mathcal{E}_{\mathrm{af}}^{\natural}, \Pi_{\mathrm{af}}, k\right)$ be an r.m.e.-datum, where $\Pi_{\mathrm{af}}=\left\{\alpha_{0}, \ldots, \alpha_{l}\right\}$. In this section, as in the previous sections, we assume that $l \geq 2$. Recall the positive integers $x_{0}, \ldots, x_{l}$ from $\S 1$. If $\alpha \in \Pi_{\mathrm{af}}$ is $\alpha_{i}$, let $x_{\alpha}:=x_{i}$ and $m_{\alpha}:=I(\alpha, \alpha) x_{\alpha} / k(\alpha)$. Let $m_{\max }:=\max \left\{m_{\alpha} \mid \alpha \in \Pi_{\mathrm{af}}\right\}$ and $\Pi_{\max }:=\left\{\alpha \in \Pi \mid m_{\alpha}=m_{\max }\right\}$. For a subset $S$ of $\Pi_{\mathrm{af}}$, let $S^{*}:=\left\{\alpha^{*} \mid \alpha \in S\right\}$. Let $\Gamma(R, G):=\Pi_{\mathrm{af}} \cup \Pi_{\max }^{*}$. The $\Gamma(R, G)$ is called the elliptic root basis (see also [SY, (2.4)]).

Following $[\mathrm{S}]$, we define the elliptic diagram (which we shall identify with the elliptic root basis $\Gamma(R, G))$ by the following rule:

(i) the vertices are in one-to-one correspondence with the elements of $\Gamma(R, G)$,

(ii) the bond and arrow between the two vertices $\alpha, \beta \in \Gamma(R, G)$ is defined in the usual manner (see $[\mathrm{K}, \S 4.8]$ ), except for the additional convention: a double dotted bond $\bigcirc===0$ if $I(\alpha, \alpha)=I(\beta, \beta)=I(\beta, \alpha)>0$.

We shall give the list of the elliptic diagrams in Appendix. Following $[\mathrm{S}]$ and $[\mathrm{ST}]$, we shall also use the following convention:

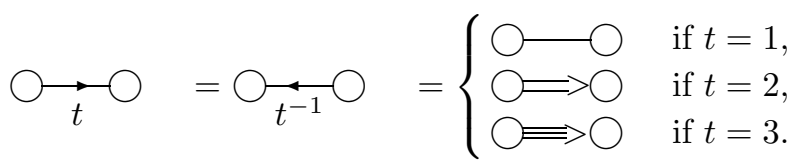

For a subset $S$ of $\Pi_{\mathrm{af}}$, let $\Gamma(R, G ; S):=\Gamma(R, G) \cap\left(S \cup S^{*}\right)$. We shall identify $\Gamma(R, G ; S)$ with the subdiagram of the elliptic diagram $\Gamma(R, G)$ formed by the vertices in $\Gamma(R, G ; S)$ and the bonds and arrows between them.

Definition 4.1. Keep the notation as above. Assume $l \geq 2$. Let $\mathfrak{g}^{\Gamma(R, G)}$ be the Lie algebra defined by the generators:

$$
h_{\sigma}\left(\sigma \in \mathcal{E}_{\mathrm{af}}^{\natural}\right), E_{\alpha}, E_{-\alpha}\left(\alpha \in \Pi_{\mathrm{af}}\right), E_{\alpha^{*}}, E_{-\alpha^{*}}\left(\alpha \in \Pi_{\max }\right)
$$

and the defining relations (see Warning below):

$(\mathrm{BS} j) \quad(1 \leq j \leq 9)\left\{\begin{array}{l}\text { The same relations as the ones among }(\mathrm{S} j) \text { expressed } \\ \text { only by the same symbols as }(4.1),\end{array}\right.$ 
$(\mathrm{BS} 10)$

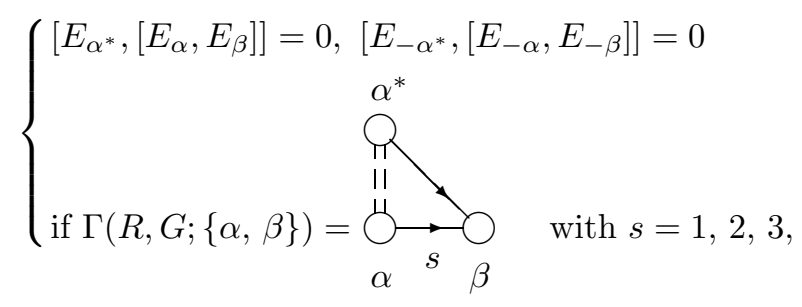

(BS11)

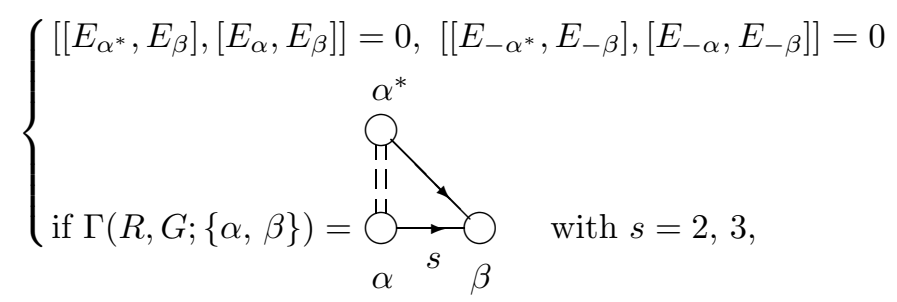

(BS12)

$$
\left\{\begin{array}{l}
{\left[\left(\operatorname{ad} E_{\beta}\right)^{-I\left(\beta^{\vee}, \alpha\right)} E_{\alpha},\left(\operatorname{ad} E_{\beta^{*}}\right)^{-I\left(\beta^{\vee}, \gamma\right)} E_{\gamma}\right]=0,} \\
{\left[\left(\operatorname{ad} E_{-\beta}\right)^{-I\left(\beta^{\vee}, \alpha\right)} E_{-\alpha},\left(\operatorname{ad} E_{-\beta^{*}}\right)^{-I\left(\beta^{\vee}, \gamma\right)} E_{-\gamma}\right]=0,} \\
\text { if } \Gamma(R, G ;\{\alpha, \beta, \gamma\})=\beta_{\alpha}^{\beta^{*}}
\end{array}\right.
$$

Warning: Here we make comments on the above $(\mathrm{BS} j)$.

(1) Needless to say, for $1 \leq j \leq 9$, $(\mathrm{BS} j)$ does not include a relation corresponding to one of $(\mathrm{S} j)$ expressed by symbols including $E_{\alpha^{*}}$ or $E_{-\alpha^{*}}$ with $\alpha \notin \Pi_{\max }$.

(2) For $5 \leq j \leq 8,(\mathrm{BS} j)$ uses the condition with a counting function $k$, but a unique counting function is associated with the elliptic diagram $\Gamma(R, G)$. The $k$ is obtained uniquely from the $\Gamma(R, G)$ in the following way:

(i) If $\Gamma(R, G ;\{\alpha, \beta\})=\bigcirc^{\alpha} \quad \stackrel{\beta}{\bigcirc}$, $k(\alpha)=k(\beta)$.
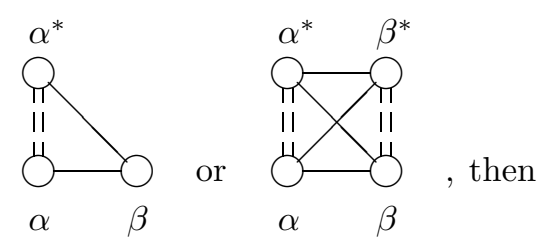

(ii) Assume that $\Gamma(R, G ;\{\alpha, \beta\})=\overbrace{\alpha} s_{\beta}$. If $s=1,2$ or 3 , then 
$k(\beta)=k(\alpha)$. If $s=2^{-1}, 3^{-1}$, then $k(\beta)=s^{-1} k(\alpha)$.

(iii) Assume that $\Gamma(R, G ;\{\alpha, \beta\})=$

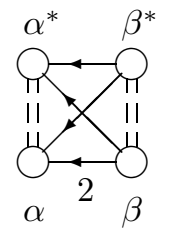
. If $I\left(\alpha^{*}, \mu\right)=0$ for all $\mu \in \Gamma(R, G) \backslash \Gamma(R, G ;\{\alpha, \beta\})$, then $k(\beta)=2 k(\alpha)$. If $I\left(\beta^{*}, \mu\right)=0$ for all $\mu \in \Gamma(R, G) \backslash \Gamma(R, G ;\{\alpha, \beta\})$, then $k(\beta)=k(\alpha)$.

(3) Denote (BS12) by $(\mathrm{BS} 12)_{\alpha, \beta, \gamma}$ to clarify $\alpha, \beta, \gamma$. Then $(\mathrm{BS} 12)_{\gamma, \beta, \alpha}$ is redundant; the relations in $(\mathrm{BS} 12)_{\gamma, \beta, \alpha}$ are obtained from $(\mathrm{BS} 12)_{\alpha, \beta, \gamma}$ and $(\mathrm{BS} j)(1 \leq j \leq 11)$. See $(2)$ of the proof of Proposition 4.2 .

We define the elements $E_{ \pm \alpha^{*}}$ of $\mathfrak{g}^{\Gamma(R, G)}$ for $\alpha \notin \Pi_{\max }$ in the following way. By seeing the list of the elliptic diagrams in Appendix, we see that there exists a unique subset $S=\left\{\beta_{1}, \ldots, \beta_{p}\right\}$ of $\Pi_{\mathrm{af}}$ such that $\beta_{p}=\alpha$, and

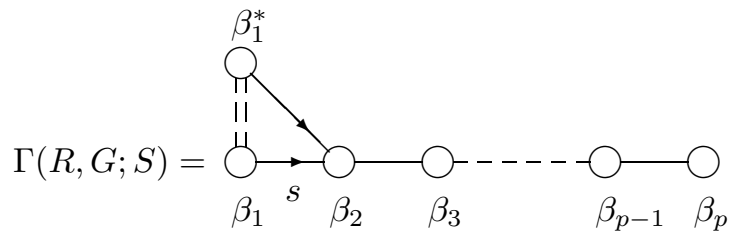

with $s=1,2^{ \pm 1}, 3^{ \pm 1}$. Then, using an induction, we define:

$$
E_{ \pm \alpha^{*}}:=(-1)^{M}\left(\operatorname{ad} E_{\mp \beta_{p-1}}\right)^{M}\left(\operatorname{ad} E_{ \pm \beta_{p-1}^{*}}\right)^{M} E_{ \pm \alpha} \in \mathfrak{g}^{\Gamma(R, G)},
$$

where $M:=-I\left(\beta_{p-1}^{\vee}, \alpha\right)$.

Recall the Lie algebra $\mathfrak{g}^{\triangle}=\mathfrak{g}^{\triangle}\left(\mathcal{E}_{\mathrm{af}}^{\natural}, \Pi_{\mathrm{af}}, k\right)$ from Definition 1.2.

Theorem 4.1. $\quad K e e p$ the notation as above. Assume $l \geq 2$. Then there exists a unique isomorphism $\Omega: \mathfrak{g}^{\triangle} \rightarrow \mathfrak{g}^{\Gamma(R, G)}$ such that $\Omega\left(h_{\sigma}\right)=h_{\sigma}\left(\sigma \in \mathcal{E}_{\mathrm{af}}^{\natural}\right)$, $\Omega\left(E_{ \pm \alpha}\right)=E_{ \pm \alpha}, \Omega\left(E_{ \pm \alpha^{*}}\right)=E_{ \pm \alpha^{*}}\left(\alpha \in \Pi_{\mathrm{af}}\right)$.

The proof shall be given at the end of this section.

Keep the notation as above. Let $S$ be a subset of $\Pi_{\mathrm{af}}$. Let $\mathfrak{g}^{\Gamma(R, G ; S)}$ be the Lie algebra defined by the generators $h_{\sigma}\left(\sigma \in \mathcal{E}_{\text {af }}^{\natural}\right), E_{ \pm \mu}(\mu \in \Gamma(R, G ; S))$ and the same defining relations as $(\mathrm{BS} j)(1 \leq j \leq 12)$. 
Lemma 4.1. Keep the notation as above. Let $\alpha, \beta \in \Pi_{\mathrm{af}}$ be such that $\alpha \neq \beta$. Let $S:=\{\alpha, \beta\}$ and $\mu \in \Gamma(R, G ; S)$. Then $C:=\left(E_{\mu}, h_{\mu^{\vee}}, E_{-\mu}\right)$ is a locally finite $\mathrm{sl}_{2}$-triple in $\mathfrak{g}^{\Gamma(R, G ; S)}$.

Proof. We may assume that $I(\alpha, \beta) \neq 0$ and $\Gamma(R, G ; S) \cap S^{*} \neq \emptyset$. Assume $\Gamma(R, G ; S)=S \cup S^{*}$. It is clear that $\mathfrak{g}^{\Gamma(R, G ; S)}=\mathfrak{g}^{(\triangle ; S)}$. The lemma for the $S$ follows from Lemma 2.2 and Proposition 2.2. So we may assume that $\Gamma(R, G ; S)=S \cup\left\{\alpha^{*}\right\}$. Moreover we may assume that $3 k(\alpha)=k(\beta)$; otherwise the lemma is clear from the relations $(\mathrm{BS} j)$. Let $\widehat{E}_{ \pm \alpha \Delta}:=n_{\alpha} n_{\beta} E_{\mp \alpha^{*}}$. Since $E_{ \pm \alpha^{*}}=n_{\beta}^{-1} n_{\alpha}^{-1} \widehat{E}_{\mp \alpha_{\Delta}}, \mathfrak{g}^{\Gamma(R, G ; S)}$ is generated by $h_{\sigma}, E_{ \pm \alpha}, E_{ \pm \beta}$ and $\widehat{E}_{ \pm \alpha_{\Delta}}$. Using the same argument as in the $D_{4}^{(3)}$ case in $\S 2.3$, we see that $\widehat{E}_{ \pm \alpha_{\Delta}}$ is locally nilpotent. Hence $E_{ \pm \alpha^{*}}$ are locally nilpotent, which completes the proof immediately.

Proposition 4.1. Let $\mu \in \Pi_{\mathrm{af}} \cup \Pi_{\mathrm{af}}^{*}$ and $C:=\left(E_{\mu}, h_{\mu^{\vee}}, E_{-\mu}\right)$. Then $C$ is a locally finite $\mathrm{sl}_{2}$-triple of $\mathfrak{g}^{\Gamma(R, G)}$.

Proof. If $\mu \in \Gamma(R, G)$, the proposition follows immediately from Lemma 4.1. Assume $\mu \notin \Gamma(R, G)$, i.e., $\mu=\alpha^{*}$ for some $\alpha \in \Pi_{\mathrm{af}} \backslash \Pi_{\max }$. Recall the definition of $E_{ \pm \mu}=E_{ \pm \alpha^{*}}$ from (4.2). By Lemmas 2.1 and 4.1 and by an induction on $p$, we see that

$$
E_{ \pm \mu}=n_{\beta_{p-1}}^{-1} n_{\beta_{p-1}^{*}} E_{ \pm \alpha},
$$

where $n_{\nu}:=n\left(\left(E_{\nu}, h_{\nu^{\vee}}, E_{-\nu}\right)\right)\left(\nu=\beta_{p-1}, \beta_{p-1}^{*}\right)$, and we also see that $E_{ \pm \mu}$ are locally nilpotent since $E_{ \pm \alpha}$ are locally nilpotent.

For $\mu \in \Pi_{\mathrm{af}} \cup \Pi_{\mathrm{af}}^{*}$, let $n_{ \pm \mu}:=n\left(\left(E_{ \pm \mu}, h_{ \pm \mu^{\vee}}, E_{\mp \mu}\right)\right) \in \operatorname{Aut}\left(\mathfrak{g}^{\Gamma(R, G)}\right)$. The $n_{ \pm \mu}$ can be defined owing to the proposition above.

Proposition 4.2. Let $S$ be a subset of $\Pi_{\mathrm{af}}$ such that $S^{*} \cap \Gamma(R, G) \neq \emptyset$ and $\Gamma(R, G ; S)$ is connected. Then there exists an epimorphism $\Omega^{(S)}: \mathfrak{g}^{(\triangle ; S)} \rightarrow$ $\mathfrak{g}^{\Gamma(R, G ; S)}$ such that $\Omega^{(S)}\left(h_{\sigma}\right)=h_{\sigma}\left(\sigma \in \mathcal{E}_{\text {af }}^{\natural}\right)$ and $\Omega^{(S)}\left(E_{ \pm \mu}\right)=E_{ \pm \mu}(\mu \in$ $\left.S \cup S^{*}\right)$.

Proof. We shall complete the proof after proving the proposition for some special $S$ 's.

(1)

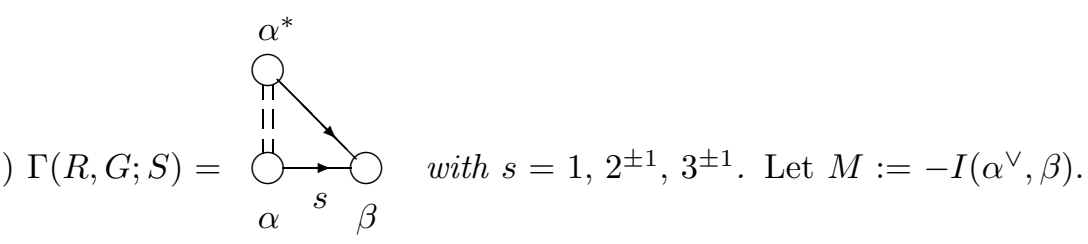


By Lemma 2.1 and Proposition 4.1, we have $n_{\mu} E_{ \pm \beta}=\left(\operatorname{ad} E_{ \pm \mu}\right)^{M} E_{ \pm \beta}$ for $\mu=\alpha, \alpha^{*}$. We can easily see that

$$
\left[E_{ \pm \alpha}, n_{\alpha^{*}} E_{ \pm \beta}\right]=0 \text { and }\left[n_{\alpha} E_{ \pm \beta}, n_{\alpha^{*}} E_{ \pm \beta}\right]=0
$$

by checking directly them for each $s$; for example, use (BS10-11) if $s \geq 1$; if $s=3^{-1}$, we have

$$
\begin{aligned}
6^{-2}\left[n_{\alpha} E_{\beta}, n_{\alpha^{*}} E_{\beta}\right] & =\left[\left(\operatorname{ad} E_{\alpha}\right)^{3} E_{\beta},\left(\operatorname{ad} E_{\alpha^{*}}\right)^{3} E_{\beta}\right]=\left(\operatorname{ad} E_{\alpha^{*}}\right)^{3}\left[\left(\operatorname{ad} E_{\alpha}\right)^{3} E_{\beta}, E_{\beta}\right] \\
& =\left(\operatorname{ad} E_{\alpha^{*}}\right)^{3}\left[\left[E_{\alpha}, E_{\beta}\right],\left(\operatorname{ad} E_{\alpha}\right)^{2} E_{\beta}\right] \\
& =0 \quad(\text { by }(\mathrm{BS} 7)) .
\end{aligned}
$$

Since $E_{ \pm \beta^{*}}=n_{\alpha}^{-1} n_{\alpha^{*}} E_{ \pm \beta}$ (see (4.3)), we have $\left[E_{ \pm \beta}, E_{ \pm \beta^{*}}\right]=0$, and by the same formula as $(2.2)$, we have $\left[E_{\mp \alpha}, E_{ \pm \beta^{*}}\right]=0$. By Lemma 2.1, we have $\left(\operatorname{ad} E_{\mp \beta}\right)^{3} E_{ \pm \beta^{*}}=0, \quad\left(\operatorname{ad} E_{\mp \beta^{*}}\right)^{3} E_{ \pm \beta}=0, \quad\left(\operatorname{ad} E_{ \pm \alpha}\right)^{M} E_{ \pm \beta^{*}}=0$ and $\left(\operatorname{ad} E_{ \pm \beta^{*}}\right)^{-I\left(\beta^{\vee}, \alpha\right)} E_{ \pm \alpha}=0$, which completes the proof of the proposition for the present $S$.

(2) $\Gamma(R, G ; S)=$

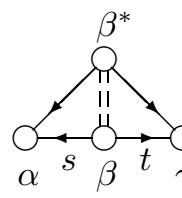
with $s, t=1,2^{ \pm 1}, 3^{ \pm 1}$. Let $\mathfrak{g}(\mu)$

be the subalgebra of $\mathfrak{g}^{\Gamma(R, G ; S)}$ generated by $E_{ \pm \mu}$ and $E_{ \pm \mu^{*}}$, where $\mu$ is $\alpha$ or $\gamma$. Using an argument similar to that in (1), we see that it suffices to show $[\mathfrak{g}(\alpha), \mathfrak{g}(\gamma)]=0$, and, by (BS12), we see that $\left[E_{ \pm \alpha}, \mathfrak{g}(\gamma)\right]=0$. Hence $\left[E_{ \pm \alpha^{*}}, \mathfrak{g}(\gamma)\right]=\left[n_{\beta}^{-1} n_{\beta^{*}} E_{ \pm \alpha}, \mathfrak{g}(\gamma)\right]=n_{\beta}^{-1} n_{\beta^{*}}\left[E_{ \pm \alpha}, \mathfrak{g}(\gamma)\right]=0$, where we can show $n_{\beta^{*}}^{-1} n_{\beta} \mathfrak{g}(\gamma)=\mathfrak{g}(\gamma)$ using $\Omega^{(\{\beta, \gamma\})}$ of this proposition and using Proposition 2.2 and Lemma 2.2.

(3) $\Gamma(R, G ; S)=$

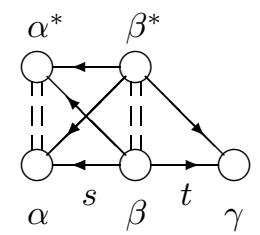
with $s, t=1,2^{ \pm 1}$. Use an argument similar to (2).

(4) $\Gamma(R, G ; S)=$

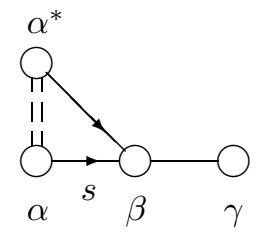
with $s=1,2^{ \pm 1}, 3^{ \pm 1}$. We first show 
that

$$
\left[E_{ \pm \beta^{*}},\left[E_{ \pm \beta}, E_{ \pm \gamma}\right]\right]=0 .
$$

Using the relations $(\mathrm{BS} j)$ and an argument similar to that in (1), we see that if $s=1,2$ or 3 , then

$$
\begin{aligned}
0 & =\left[\left[\left[E_{\alpha},\left[E_{\alpha^{*}}, E_{\beta}\right]\right], E_{\gamma}\right], E_{\beta}\right] \\
& =\left[\left[E_{\alpha},\left[E_{\alpha^{*}},\left[E_{\beta}, E_{\gamma}\right]\right]\right], E_{\beta}\right] \\
& =\left[\left[E_{\alpha}, E_{\beta}\right],\left[E_{\alpha^{*}},\left[E_{\beta}, E_{\gamma}\right]\right]\right]+\left[E_{\alpha},\left[\left[E_{\alpha *}, E_{\beta}\right],\left[E_{\beta}, E_{\gamma}\right]\right]\right] \\
& =\left[\left[E_{\alpha}, E_{\beta}\right],\left[\left[E_{\alpha^{*}}, E_{\beta}\right], E_{\gamma}\right]\right]+\left[\left[E_{\alpha *}, E_{\beta}\right],\left[\left[E_{\alpha}, E_{\beta}\right], E_{\gamma}\right]\right] \\
& =2\left[\left[E_{\alpha^{*}}, E_{\beta}\right],\left[\left[E_{\alpha}, E_{\beta}\right], E_{\gamma}\right]\right] \quad(\text { by }(\mathrm{BS} 11)) \\
& =2\left[n_{\alpha^{*}} E_{\beta},\left[n_{\alpha} E_{\beta}, E_{\gamma}\right]\right] \\
& =2 n_{\alpha}\left[E_{\beta^{*}},\left[E_{\beta}, E_{\gamma}\right]\right]
\end{aligned}
$$

and that if $s^{-1}=2$ or 3 , then letting $c:=s^{-1}$, we have:

$$
\begin{aligned}
0 & =\left(\operatorname{ad} E_{\alpha}\right)^{c-1}\left(\operatorname{ad} E_{\alpha^{*}}\right)^{c} n_{\beta}\left[E_{\alpha}, E_{\gamma}\right] \\
& =\left(\operatorname{ad} E_{\alpha}\right)^{c-1}\left(\operatorname{ad} E_{\alpha^{*}}\right)^{c}\left[\left[E_{\beta}, E_{\alpha}\right],\left[E_{\beta}, E_{\gamma}\right]\right] \\
& =\left(\operatorname{ad} E_{\alpha}\right)^{c-1}\left(\operatorname{ad} E_{\alpha^{*}}\right)^{c}\left[E_{\beta},\left[\left[E_{\beta}, E_{\alpha}\right], E_{\gamma}\right]\right] \\
& =-\left[\left(\operatorname{ad} E_{\alpha^{*}}\right)^{c} E_{\beta},\left[\left(\operatorname{ad} E_{\alpha}\right)^{c} E_{\beta}, E_{\gamma}\right]\right] \\
& =-(c !)^{2}\left[n_{\alpha^{*}} E_{\beta},\left[n_{\alpha} E_{\beta}, E_{\gamma}\right]\right] \\
& =-(c !)^{2} n_{\alpha}\left[E_{\beta^{*}},\left[E_{\beta}, E_{\gamma}\right]\right] .
\end{aligned}
$$

Similarly we have $\left[E_{-\beta^{*}},\left[E_{-\beta}, E_{-\gamma}\right]\right]=0$. Thus we have (4.4). Using (4.4) and arguments similar to those in (1) and (3), we get the proposition for the present $S$.

(5) $\Gamma(R, G ; S)=$

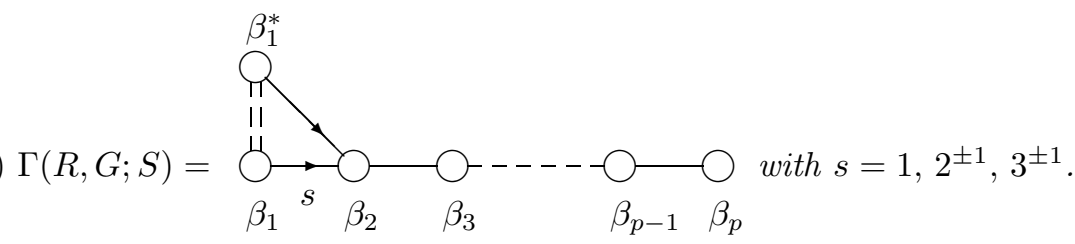

This can be done by iterating the argument in (4).

(6) We can complete the proof of the proposition for a general $S$ easily by using the list of the elliptic diagrams in Appendix and using the arguments in (1) $-(5)$. 
Proof of Theorem 4.1. It is clear from Proposition 4.2 that the homomorphism $\Omega$ of the statement exists. Recall Proposition 3.3. Notice that no non-zero weight space of $\mathfrak{g}^{\triangle}$ contains an element corresponding to a relation in (BS $j)(10 \leq j \leq 12)$. Then there exists the homomorphism $\Xi: \mathfrak{g}^{\Gamma(R, G)} \rightarrow \mathfrak{g}^{\triangle}$ such that $\Xi\left(h_{\sigma}\right)=h_{\sigma}\left(\sigma \in \mathcal{E}_{\text {af }}^{\natural}\right)$ and $\Xi\left(E_{ \pm \mu}\right)=E_{ \pm \mu}(\mu \in \Gamma(R, G))$. Using the relation (S5) and an argument similar to that for (4.3), we see that $\Xi\left(E_{ \pm \nu}\right)=E_{ \pm \nu}\left(\nu \in \Pi_{\text {af }}^{*} \backslash \Gamma(R, G)\right)$. Clearly $\Xi$ is the inverse map of $\Omega$.

\section{Appendix}

\section{Definition of an r.m.e.-root System}

Keep the notation as in the fourth paragraph of Introduction. For a subspace $\mathcal{X}$ of $\mathcal{E}$, let $\mathcal{X}^{\perp}$ be the subspace of $\mathcal{E}$ of the elements $y$ satisfying the condition that $I(x, y)=0$ for all $x \in \mathcal{X}$. For a subset $S$ of $\mathcal{E}$ and an additive subgroup $\mathbf{A}$ of $\mathbb{C}$, denote by $Q_{\mathbf{A}}(S)$ the $\mathbf{A}$ submodule of $\mathcal{E}$ generated by the elements of $S$.

Let $R$ be a subset of $\mathcal{E}$. Assume that $I(x, x) \neq 0$ for any $x \in R$. We say that $R$ is an elliptic root system if:

$(\mathrm{r} 1) \operatorname{dim}_{\mathbb{C}} Q_{\mathbb{C}}(R)=l+2=\operatorname{rank}_{\mathbb{Z}} Q_{\mathbb{Z}}(R)$ and $\operatorname{dim}_{\mathbb{C}} Q_{\mathbb{C}}(R) \cap Q_{\mathbb{C}}(R)^{\perp}=2$,

$(\mathrm{r} 2) I\left(x^{\vee}, y\right) \in \mathbb{Z}$ for $x, y \in R$; and $\frac{I(\sigma, \sigma)}{I(x, x)} \in \mathbb{R}_{+}$for all $x \in R$ and all $\sigma \in Q_{\mathbb{Z}}(R)$,

(r3) $s_{x}(R)=R$ for all $x \in R$,

(r4) There exists no subspace $\mathcal{X}$ of $\mathcal{E}$ such that $(\mathcal{X} \cap R) \cup\left(\mathcal{X}^{\perp} \cap R\right)=R$, $(\mathcal{X} \cap R) \neq \emptyset$ and $\left(\mathcal{X}^{\perp} \cap R\right) \neq \emptyset$.

Here we call $l$ the rank.

Let $R$ be an elliptic root system. A one dimensional subspace $G$ of $Q_{\mathbb{C}}(R) \cap$ $Q_{\mathbb{C}}(R)^{\perp}$ is called a marking if $Q_{\mathbb{Q}}(R) \cap G \neq\{0\}$. The pair $(R, G)$ of such $R$ and $G$ is called a marked elliptic root system.

We say that a marked elliptic root system $(R, G)$ is reduced if $2 y-x \notin G$ for all $x, y \in R$. In this paper, the terminology "a reduced marked elliptic root system" has been abbreviated to an r.m.e.-root-system.

An r.m.e.-root-system $(R, G)$ is called simply-laced if $\Gamma(R, G)$ is $A_{l}^{(1,1)}$ with $l \geq 2, D_{l}^{(1,1)}$ with $l \geq 4$ or $E_{l}^{(1,1)}$ with $l=6,7,8$ (see the table below).

Table of the elliptic diagrams with $l \geq 2$

Convention: In the following, we use the convention below: 


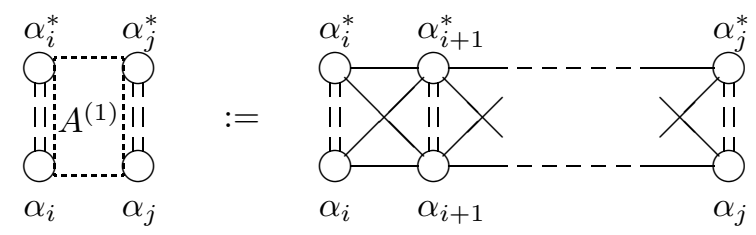

$(0 \leq i \leq j \leq l)$.

$A_{l}^{(1,1)}(l \geq 2)$

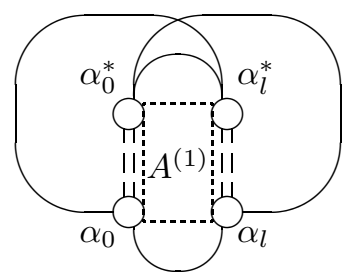

$B_{l}^{(1,1)}(l \geq 3)$

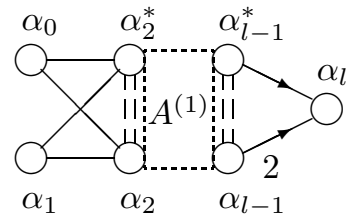

$B_{l}^{(2,2)}(l \geq 2)$

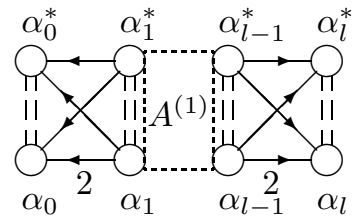

$C_{l}^{(2,1)}(l \geq 3)$

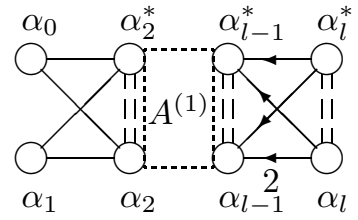

$B_{l}^{(1,2)}(l \geq 3)$

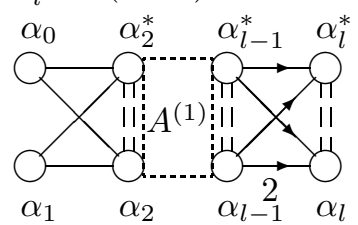

$C_{l}^{(1,1)}(l \geq 2)$

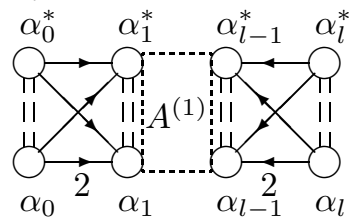

$C_{l}^{(2,2)}(l \geq 3)$

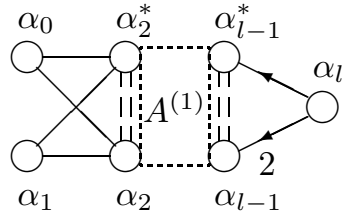

$B_{l}^{(2,1)}(l \geq 2)$

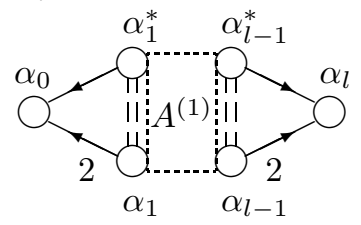

$C_{l}^{(1,2)}(l \geq 2)$

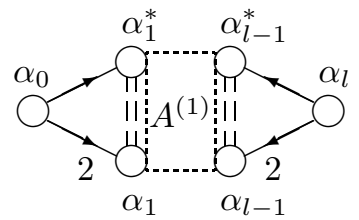

$B_{l}^{(2,2) *}(l \geq 2)$

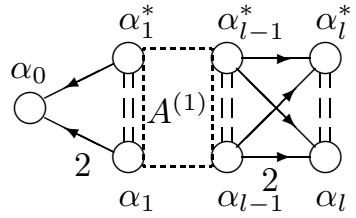



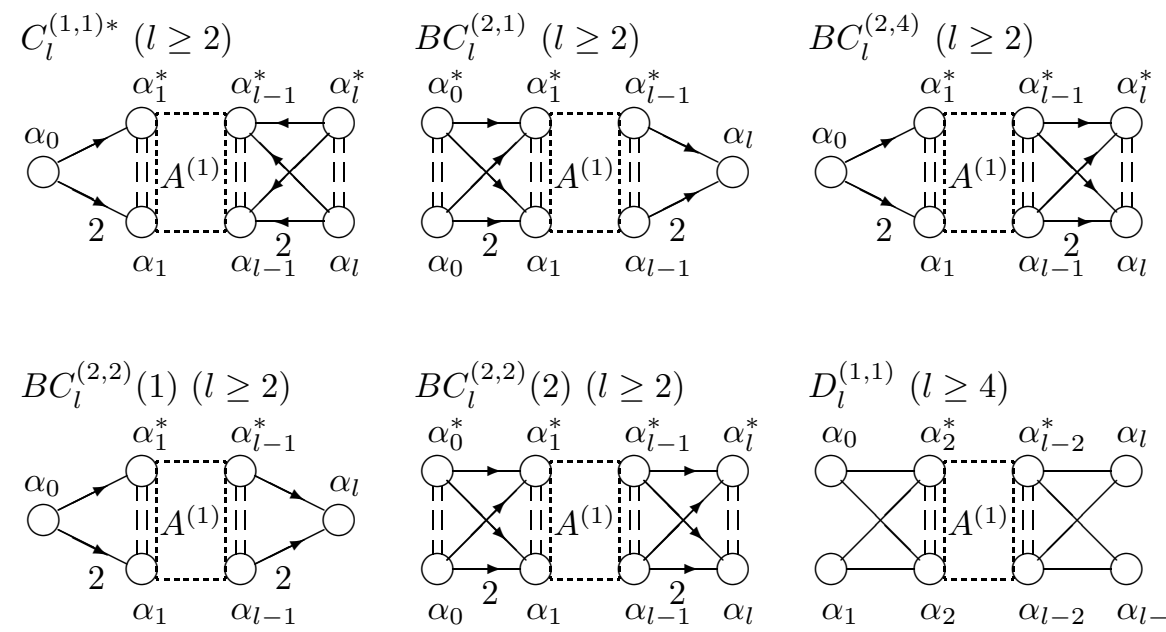

$D_{l}^{(1,1)}(l \geq 4)$
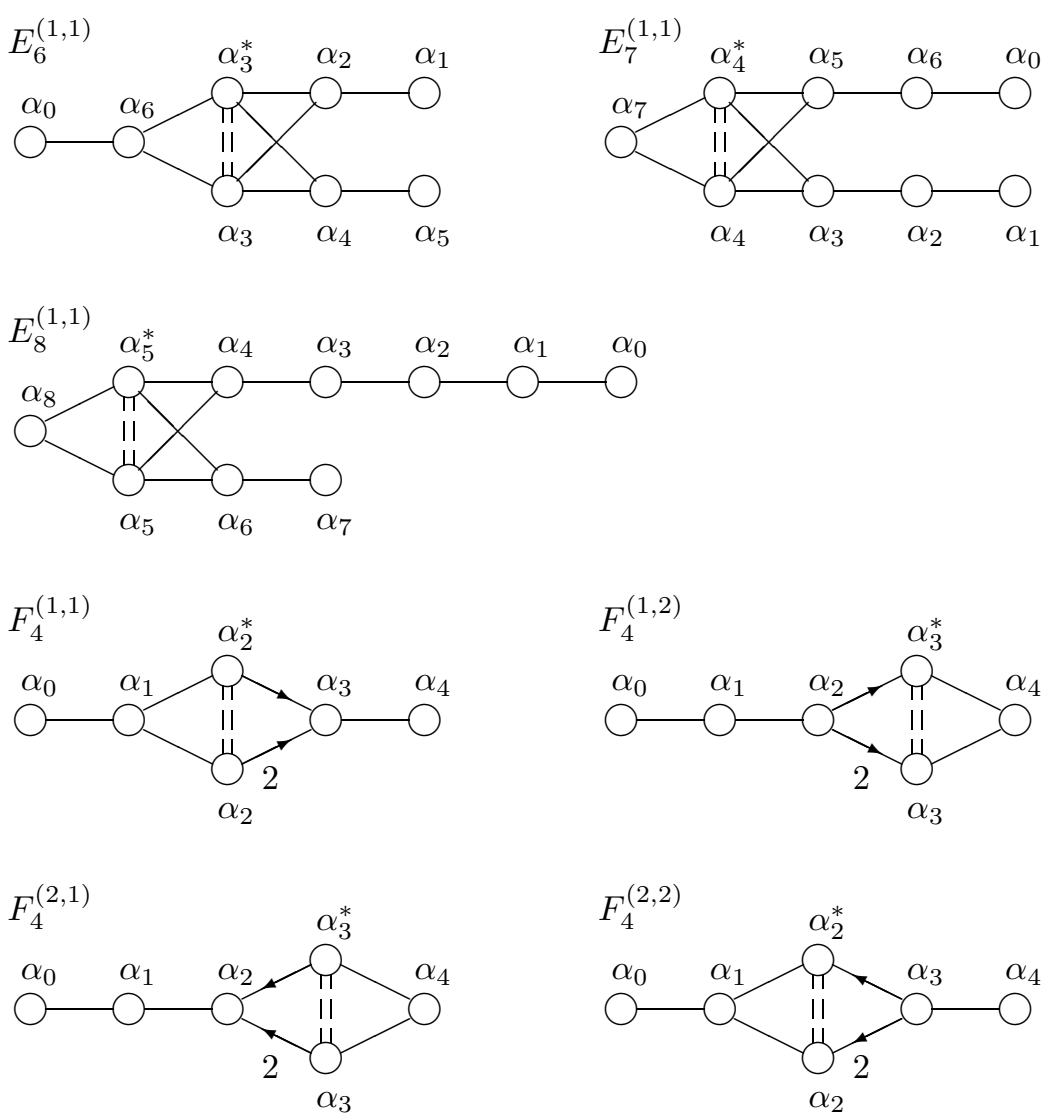

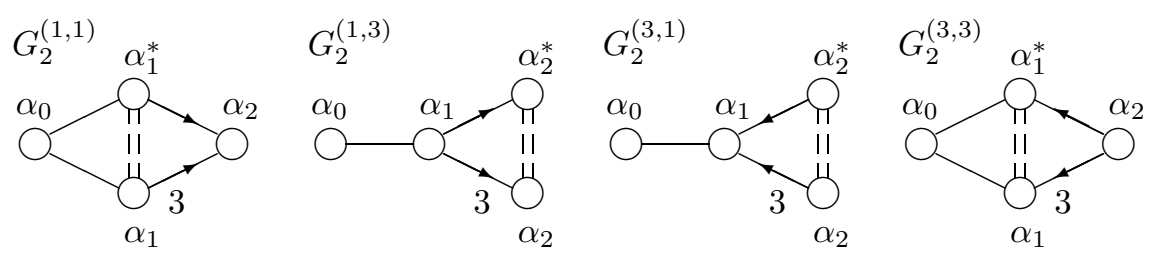

Table of the elliptic diagrams with $l=1$
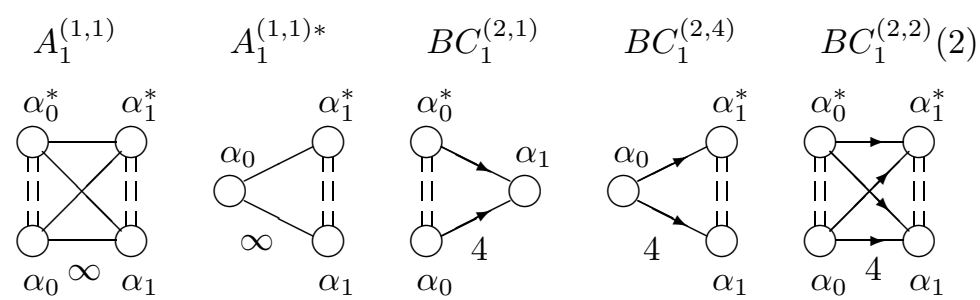

Here

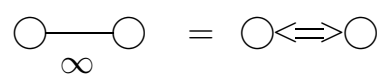

and
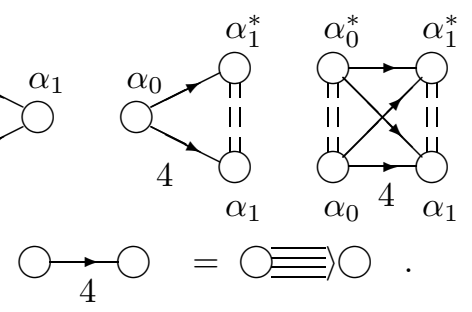

\section{Acknowledgement}

The author would like to express his thanks to Professor Kyoji Saito, who made valuable comments on the paper and offered the suggestion that he should also give Theorem 4.1. The author decided to add Theorem 3.2 under the inspiration from K. Saito's lecture of Tokyo University in April of 2002.

\section{References}

[K] Kac, V.G., Infinite dimensional Lie algebras (3rd ed.), Cambridge Univ. Press, Cambridge, 1990.

[M1] Miki, K., Toroidal braid group action and an automorphism of toroidal algebra $U_{q}\left(s_{n+1, t o l}\right)(n \geq 2)$, Lett. Math. Phys., 47 (1999), 365-378.

[M2] Luantum toroidal algebra $U_{q}\left(s l_{2, \text { tol }}\right)$ and $R$ matrices, J. Math. Phys., 42 (2001), 2293-2308.

[P] Pollmann, U., Realisation der biaffine Wurzelsysteme von Saito in Lie-Algebren, Hamburger Beiträge zur Mathematik, Heft, 29 (1994).

[S] Saito, K., Extended affine root systems I (Coxeter Transformations), Publ. RIMS, Kyoto Univ., 31 (1985), 75-179.

[ST] Saito, K. and Takebayashi, T., Extended affine root systems III (Elliptic Weyl Groups), Publ. RIMS, Kyoto Univ., 33 (1997), 301-329.

[SY] Saito, K. and Yoshii, D., Extended affine root systems IV (Simply-Laced Elliptic Lie Algebras), Publ. RIMS, Kyoto Univ., 36 (2000), 385-421.

[Y] Yoshii, D., Elliptic Lie algebras (Inhomogeneous Cases), Preprint, RIMS-1308 (2000). 